\title{
TCTEX1D2 mutations underlie Jeune asphyxiating thoracic dystrophy with impaired retrograde intraflagellar transport
}

Miriam Schmidts ${ }^{1,2,3,4, \star}$, Yuqing Hou ${ }^{5, \star}$, Claudio R. Cortés ${ }^{6}$, Dorus A. Mans ${ }^{2,3}$, Celine Huber ${ }^{7}$, Karsten Boldt $^{8}$, Mitali Patel ${ }^{1}$, Jeroen van Reeuwijk ${ }^{2,3}$, Jean-Marc Plaza9 , Sylvia E.C. van Beersum²,3, Zhi Min Yap ${ }^{1}$, Stef J.F. Letteboer ${ }^{2,3}$, S Paige Taylor ${ }^{10}$, Warren Herridge ${ }^{11}$, Colin A. Johnson ${ }^{11}$, Peter J. Scambler ${ }^{12}$, Marius Ueffing ${ }^{8}$, Hulya Kayserili13,14, Deborah Krakow ${ }^{10}$, Stephen M. King ${ }^{15}$, UK10K ${ }^{\dagger}$, Philip L. Beales ${ }^{1,16}$, Lihadh Al-Gazali ${ }^{17}$, Carol Wicking6 ${ }^{6}$, Valerie Cormier-Daire ${ }^{7}$, Ronald Roepman ${ }^{2,3}$, Hannah M. Mitchison ${ }^{1, \star \star}$, George B. Witman ${ }^{5, \star \star}$

The analysis of individuals with ciliary chondrodysplasias can shed light on sensitive mechanisms controlling ciliogenesis and cell signalling that are essential to embryonic development and survival. Here we identify TCTEX1D2 mutations causing Jeune asphyxiating thoracic dystrophy with partially penetrant inheritance. Loss of TCTEX1D2 impairs retrograde intraflagellar transport (IFT) in humans and the protist Chlamydomonas, accompanied by destabilization of the retrograde IFT dynein motor. We thus define TCTEX1D2 as an integral component of the evolutionarily conserved retrograde IFT machinery. In complex with several IFT dynein light chains, it is required for correct vertebrate skeletal formation but may be functionally redundant under certain conditions.

\footnotetext{
${ }^{1}$ Genetics and Genomic Medicine Programme, University College London (UCL), Institute of Child Health, 30 Guilford Street, London WC1N 1EH, UK.

${ }^{2}$ Department of Human Genetics, Radboud University Medical Center, 6525 GA Nijmegen, The Netherlands. ${ }^{3}$ Radboud Institute for Molecular Life Sciences, Radboud University Medical Center, 6525 GA Nijmegen, The Netherlands. ${ }^{4}$ Center for Pediatrics and Adolescent Medicine, University Hospital Freiburg, Mathildenstrasse 1, 79112 Freiburg, Germany. ${ }^{5}$ Department of Cell and Developmental Biology, University of Massachusetts Medical School, Worcester, Massachusetts 01655, USA. ${ }^{6}$ Institute for Molecular Bioscience, The University of Queensland, St Lucia, Queensland 4072, Australia. ${ }^{7}$ INSERM UMR_1163, Département de génétique, Institut Imagine, Université Paris Descartes Sorbonne Paris Cité, Hôpital Necker-Enfants Malades, Assistance Publique-Hôpitaux de Paris, Paris 75015, France. ${ }^{8}$ Division of Experimental Ophthalmology and Medical Proteome Center, Center of Ophthalmology, University of Tübingen, Tübingen 72074, Germany. ${ }^{9}$ Plateforme de Bioinformatique, Institut Imagine, Université Paris Descartes, Paris 75015, France. ${ }^{10}$ Departments of Orthopaedic Surgery and Human Genetics, David Geffen School of Medicine at UCLA, Los Angeles 90095, California, USA. ${ }^{11}$ Section of Ophthalmology and Neuroscience, Leeds Institutes of Molecular Medicine, University of Leeds, Leeds LS9 7TF, UK. ${ }^{12}$ Developmental Biology and Cancer Programme, University College London (UCL), Institute of Child Health, 30 Guilford Street, London WC1N 1EH, UK. ${ }^{13}$ Medical Genetics Department, Istanbul Medical Faculty, Istanbul University, 34093 Istanbul, Turkey. ${ }^{14}$ Medical Genetics Department, Koc University School of Medicine, 34010 Istanbul, Turkey. ${ }^{15}$ Department of Molecular Biology and Biophysics and Institute for Systems Genomics, University of Connecticut Health Center, Farmington, Connecticut 06030, USA. ${ }^{16}$ Centre for Translational Genomics-GOSgene, UCL Institute of Child Health, London WC1N 1EH, UK. ${ }^{17}$ Department of Pediatrics, College of Medicine and Health Sciences, United Arab Emirates University, PO Box 17666, Al Ain, United Arab Emirates. ${ }^{\star}$ These authors contributed equally to this work. ${ }^{\star \star}$ These authors jointly supervised the work. $\dagger$ A full list of consortium members appears at the end of the paper. Correspondence and requests for materials should be addressed to H.M.M. (email: h.mitchison@ucl.ac.uk)
} 
T he malfunction of primary cilia, conserved signalling organelles present on the surface of most cells, has emerged as the cause of a growing number of severe congenital developmental defects ${ }^{1,2}$. Intraflagellar transport (IFT), a highly conserved process required for ciliary growth and signalling, is powered by motors attached to IFT complexes $\mathrm{A}$ and $\mathrm{B}$. The kinesin-2 motor transports cargo along ciliary microtubules towards the ciliary tip (anterograde IFT). IFT complexes and other cargo are returned to the base of the cilium (retrograde IFT) by a specialized cytoplasmic dynein ${ }^{3}$, termed dynein 2 in vertebrates and dynein $1 \mathrm{~b}$ in Chlamydomonas; (hereafter referred to as 'IFT dynein'). Chlamydomonas has been a key organism for elucidating the molecular and mechanistic basis of IFT. The known subunits of Chlamydomonas IFT dynein are the homodimer-forming heavy chain DHC1b, intermediate chains D1bIC1 and D1bIC2 (also known as FAP163 and FAP133, respectively ${ }^{4}$ ), light-intermediate chain D1bLIC and the light-chain LC8. Human IFT dynein contains the homologues of these proteins, DYNC2H1, WDR60, WDR34, DYNC2LI1 and DYNLL1/DYNLL2 (both of which are LC8 homologues), and probably additional subunits ${ }^{4-10}$. However, the regulation, cargo interactions and exact composition of IFT dynein have remained relatively elusive.

Primary cilia and IFT are required for vertebrate hedgehog signalling, an important regulator of skeletogenesis ${ }^{11}$. Mutations in several IFT dynein components cause short-rib polydactyly syndromes (SRPS) and Jeune asphyxiating thoracic dystrophy (JATD; Jeune syndrome), a group of autosomal recessively inherited, genetically heterogeneous ciliary chondrodysplasias with overlapping phenotypic features ${ }^{12-16}$. The spectrum of disease phenotypes varies in affected individuals but hallmarks include short ribs and narrow thorax, short limbs, with sporadic polydactyly and extraskeletal disease including kidney, liver, eye, heart and brain defects. The underlying genetic basis of these skeletal ciliopathies also overlaps, with JATD at the milder end of the JATD/SRPS disease spectrum ${ }^{17}$. To date, mutations in 10 genes have been shown to cause Jeune syndrome, eight leading to classic JATD with ciliogenesis defects due to IFT dysfunction; these encode heavy and intermediate IFT dynein subunits DYNC2H1, WDR34 and WDR60 (refs 12-16), IFT complex B components IFT80 and IFT172 (refs 18,20), and IFT complex A components WDR19/IFT144, TTC21B/IFT139 and IFT140 (refs 21-23). Two encode the centriole-associated proteins CEP120 and CSPP1 (Jeune variants), which are important for ciliary assembly or function ${ }^{19,24}$. Disease-causing mutations are considered hypomorphic since no individuals with SRPS or JATD were previously shown to carry biallelic loss-of-function mutations ${ }^{13,17}$, and homozygosity for 'null' alleles is embryonic lethal in mouse models around midgestation ${ }^{25,26}$.

Here we report biallelic loss-of-function mutations causing JATD in the gene encoding TCTEX1D2, an IFT dynein light chain distinct from DYNLL1/DYNLL2 (LC8). Unusually for the SRPS/JATD spectrum, affected individuals all carry biallelic 'null' alleles where complete loss of protein function is predicted. Furthermore, the disease phenotype appears incompletely penetrant. Affinity proteomics indicates that TCTEX1D2/Tctex $2 \mathrm{~b}$ is an integral component of IFT dynein. We demonstrate a retrograde IFT defect in TCTEX1D2-deficient human fibroblasts and Tctex2b-deficient Chlamydomonas cells, and find that IFT dynein is partially destabilized by loss of Tctex $2 \mathrm{~b}$ in Chlamydomonas. Compared with mutations in other IFT dynein components, TCTEX1D2/Tctex2b loss in human, zebrafish and Chlamydomonas has a modest effect on retrograde IFT, likely explaining the partially penetrant nature of human TCTEX1D2 mutations.

\section{Results}

Variants in TCTEX1D2 are associated with JATD. Wholeexome sequencing of 69 individuals from 60 families clinically diagnosed with JATD identified a homozygous consensus splice variant $(c .113+2 \mathrm{C}>\mathrm{G})$ in TCTEX1D2 (NM_152773.4, encoding a dynein light chain) in individual UCL82 II.1 from a consanguineous Turkish family (Supplementary Fig. 1a). Reverse transcription PCR (RT-PCR) on RNA derived from fibroblasts of UCL82 II.1 detected no TCTEX1D2 transcript, suggesting nonsense-mediated decay (NMD) of the mutant transcript (Supplementary Fig. 2a). All primers used for human genetic analysis are listed in Supplementary Table 1. Exome copy number variant analysis revealed a $>10-\mathrm{kb}$ homozygous deletion in two affected siblings (UCL4 II.6 and II.8) from a consanguineous Arabic family that removes exon 1-2 of TCTEX1D2 including the start codon, indicating a complete loss-of-function allele (c.(?_- 142)_247+ ?del) (Supplementary Fig. 3). No other likely disease-causing variants in known JATD/SRPS-causing genes or other known ciliary components were detected in these two siblings. The deletion also removes exon $2-5$ of neighbouring TM4SF19 encoding a protein reportedly involved in pancreatic development but unlikely to be involved in JATD (Supplementary Fig. 3). Reverse transcription PCR on RNA derived from blood lymphocytes of individuals UCL4 II.6 and II.8 detected no TCTEX1D2 transcript, indicating likely NMD of the mutant transcript (Supplementary Fig. 2b), and no transcripts initiated at the TM4SF19 start codon continuing into TCTEX1D2 downstream of the deletion either. Analysis of a further 154 JATD/SRPS exomes and Sanger sequencing of TCTEX1D2 in 69 additional JATD/SRPS cases, previously excluded for mutations in known JATD and SRPS genes, detected compound-heterozygous variants in TCTEX1D2 in individual INS II.1 from a non-consanguineous French family comprising a nonsense (c.262C > T; p.Arg88 ${ }^{\star}$ ) and a deletioninsertion frameshift alteration (c.100delinsCT; p.Val34Leufs ${ }^{\star} 12$; Supplementary Fig. 1b).

The TCTEX1D2 c.113 + 2C > G, c.262C > T and c.100delinsCT variants are absent from the dbSNP, 1,000 Genomes and EVC databases. The exon 1-2 deletion is absent from 500 exomes from the UK10K project and 100 Bedouin control chromosomes assessed by Sanger sequencing. The c.113+2C > G, c.262C > T and c.100delinsCT variants segregated with the disease phenotype in affected families as expected (Supplementary Fig. 1). However, in family UCL4, the exon 1-2 deletion was detected not only in a third affected individual who died at 2 months due to respiratory insufficiency (UCL4 II.9) but also in two siblings (UCL4 II.1 and II.5) for whom no clinical signs of JATD had been documented (Fig. 1a and Supplementary Fig. 1c,d). When reassessed clinically with a full X-ray exam, both UCL4 II.1 and II.5 showed mild brachydactyly and slightly shortened lower limb distal segments; they are both shorter in stature than their siblings that do not carry the deletion, and one also had pectus carinatum as a child. However, in contrast to the other five individuals documented here as affected with typical clinical features of JATD (short horizontal ribs, narrow thorax, trident acetabulum with spurs and polydactyly; Fig. 2 and Table 1), no specific radiological signs of JATD/SRPS were found in UCL4 II.1 and II.5 (Supplementary Fig. 4). Thus, while their mild brachydactyly and short stature could represent a very mild JATD phenotype, or radiological diagnostic criteria for JATD might have been fulfilled in childhood, we have no clinical or radiological proof for JATD. Brachydactyly and short stature can occur with JATD but are not specific for the condition. We therefore suggest that the phenotype is not fully penetrant in this family, with the caveat that we cannot definitively exclude an extremely mild expression of the JATD phenotype in the two seemingly unaffected individuals harbouring TCTEX1D2 mutations. 
a

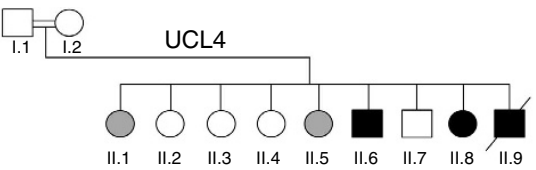

$\begin{array}{llllllllllll}\text { M } & \text { I.1 } & \text { I.2 } & \text { II.1 } & \text { II.2 } & \text { II.3 } & \text { II.4 } & \text { II.5 } & \text { II.6 } & \text { II.7 } & \text { II.8 } & \text { II.9 }\end{array}$

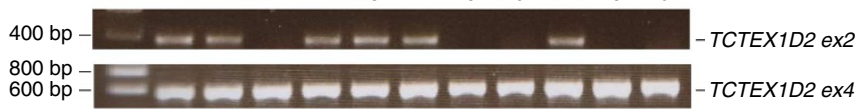

b

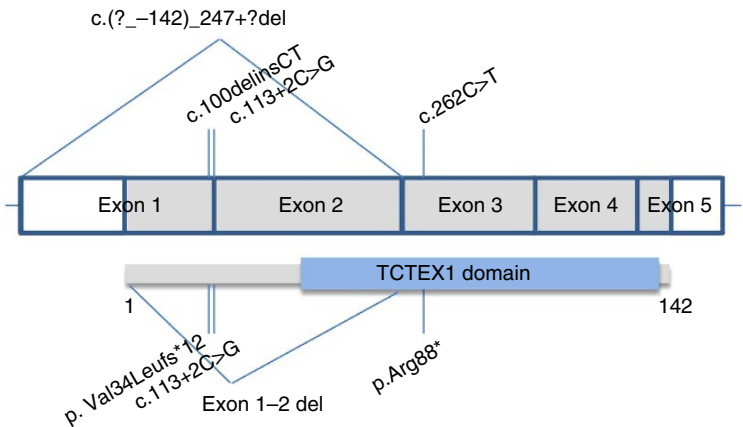

Figure 1 | TCTEX1D2 deletion in UCL4 and location of identified variants in TCTEX1D2 protein structure. (a) The absence of TCTEX1D2 exon 1 and 2 in family UCL4 is visualized by PCR of genomic DNA samples from members of the UCL4 pedigree. TCTEX1D2 exon 4 primers verify the presence of the gene in samples, but TCTEX1D2 exon 2 primers do not amplify in some individuals. Children carrying the homozygous exon 1-2 TCTEX1D2 deletion are marked in black (diagnosed with JATD) or grey (two siblings who were not diagnosed with JATD). The strikethrough indicates death at 2 months of age; double line indicates consanguineous marriage. See also Supplementary Fig. 1. (b) Human TCTEX1D2 (shown above, white boxes indicate untranslated regions (UTR)) consists of five exons encoding a 142 amino-acid protein (shown below) with a C-terminal TCTEX1 domain (blue box). The location of the four identified TCTEX1D2 mutations is shown in the gene (above). Their corresponding location in the protein (below) shows the TCTEX1 domain will be at least partially lost for all variants identified in individuals with JATD.

We examined this further by single-nucleotide polymorphismbased genome-wide linkage analysis in all members of UCL4. A high penetrance recessive model coding UCL4 II.1 and II.5 as unknown identified five homozygous linked regions all with a logarithm of odds (LOD) score of 2.9, with TCTEX1D2 in the second largest interval (Supplementary Table 2). Cross-reference to the exome-sequencing data detected the TCTEX1D2 deletion and just one other homozygous variant shared between UCL4 II.6 and II. 8 in these intervals. The latter was in an untranslated pseudogene TBC1D3P2 with a minor allele frequency of 1 in 500 in our in-house control database, suggesting it is not causative for JATD. Considering UCL4 II. 1 and II.5 as affected under a reduced penetrance model (penetrance set at 0.6) generated three linked regions including one with a LOD score 3.9 across TCTEX1D2, with no homozygous variants shared between UCL4 II.6 and II.8 in the other intervals (Supplementary Table 3). Considering UCL4 II.1 and II.5 as unaffected found no linkage to homozygous variants shared between UCL4 II.6 and II.8 except the TBC1D3P2 pseudogene variant (LOD score 2.9; Supplementary Table 4). Finally, considering UCL4 II.1 and II.5 as affected under the high penetrance model identified just one linked region, a 9.4-Mb locus containing TCTEX1D2 with a LOD score of 4.1 (Supplementary Table 5). Thus, along with the expected segregation in two independent families, linkage modelling supports TCTEX1D2 mutations as disease-causing in family UCL4.
Functional predictions and evidence of NMD suggest that all four TCTEX1D2 variants identified are loss-of-function mutations affecting the conserved TCTEX1 domain of the protein (Fig. 1b). The questionable disease status in individuals UCL4 II.1 and II.5 suggests incomplete penetrance in this family, an inheritance pattern not previously described for JATD.

Knockdown of tctex1d2 causes a typical ciliopathy phenotype. TCTEX1D2 function was not previously investigated in vertebrates and we tested for potential redundancy of the orthologous gene in zebrafish using oligonucleotide antisense morpholinos to abrogate transcription. Morpholino oligomers have been used widely to provide models for Jeune syndrome by transiently knocking down the expression of the orthologous zebrafish genes $^{18-20}$. This approach has been criticized for its inability to reliably discriminate between specific and non-specific effects such as developmental delay and cardiac oedema ${ }^{27,28}$. To increase reliability, we established that human (NP_689986.2) and zebrafish (XP_685487.3) TCTEX1D2 proteins are highly homologous, being $60 \%$ identical at $80 \%$ BLAST coverage. We separately tested two different splice-blocking morpholinos and focussed on phenotypic features described in JATD zebrafish mutants as well as morphants ${ }^{29-33}$.

Both morpholinos yielded identical results, producing a dosedependent typical ciliopathy phenotype ${ }^{18,20}$, with ventrally curved body axis, hydrocephalus, abnormal otoliths and small eyes (Fig. 3a-h). Loss of transcript was confirmed by RT-PCR (Supplementary Fig. 5 and Supplementary Table 1). The few embryos surviving to 4 days post fertilization displayed severe generalized oedema and pronephric cysts (Fig. 3c,g). Embryos also showed defects of the craniofacial cartilage as visualized by alcian blue staining (Fig. 3i,j,m,n), which were comparable to those observed in other ciliary chondrodysplasia zebrafish models such as ift80, ift172 and cspp1 (refs 18-20). Immunofluorescence analysis revealed shorter cilia in the pronephric duct at $24 \mathrm{~h}$ post fertilization (h.p.f.) in tctex $1 d 2$ morphant embryos compared with controls (Fig. 3k,o,l,p); however, this difference was no longer observed at 48 h.p.f. (not shown). This could reflect delayed ciliogenesis in the morphants or could result from general mild developmental delay. Cilia in the neural tube appeared normal (Supplementary Fig. 6).

Loss of TCTEX1D2 causes a retrograde IFT defect. We proceeded to investigate skin fibroblasts from individual UCL82 II.1 for defects in cilia architecture and IFT disturbance. While the percentage of ciliated cells was modest but significantly lower for TCTEX1D2 mutant fibroblasts compared with controls (Fig. 4e), no significant difference in ciliary length was observed after $24-\mathrm{h}$ serum starvation, in contrast to the cilia shortening reported in individuals with mutations in other JATD/SRPS genes ${ }^{12,19}$ (Fig. 4f). However, there was marked accumulation of the IFT-particle protein IFT 88 in $\sim 35 \%$ of the ciliary tips of TCTEX1D2-deficient fibroblasts, compared with $<10 \%$ of control fibroblasts (Fig. 4a-d). Cells from an individual previously reported to carry DYNC2H1 mutations ${ }^{34}$ showed a very similar pattern, which indicates a retrograde IFT defect (Fig. 4d).

Chlamydomonas Tctex2b is a light chain of IFT dynein. We further investigated the role in IFT of Tctex $2 \mathrm{~b}$, the Chlamydomonas homologue of TCTEX1D2 (49\% identical at 80\% BLAST coverage; human to Chlamydomonas reciprocal best match BLAST 9e-36). To determine whether Tctex $2 \mathrm{~b}$ is a subunit of IFT dynein in Chlamydomonas, we used a new strain expressing D1bIC2 (homologue of human IFT dynein intermediate chain WDR34) fused to the haemagglutin-epitope tag (HA). This was 

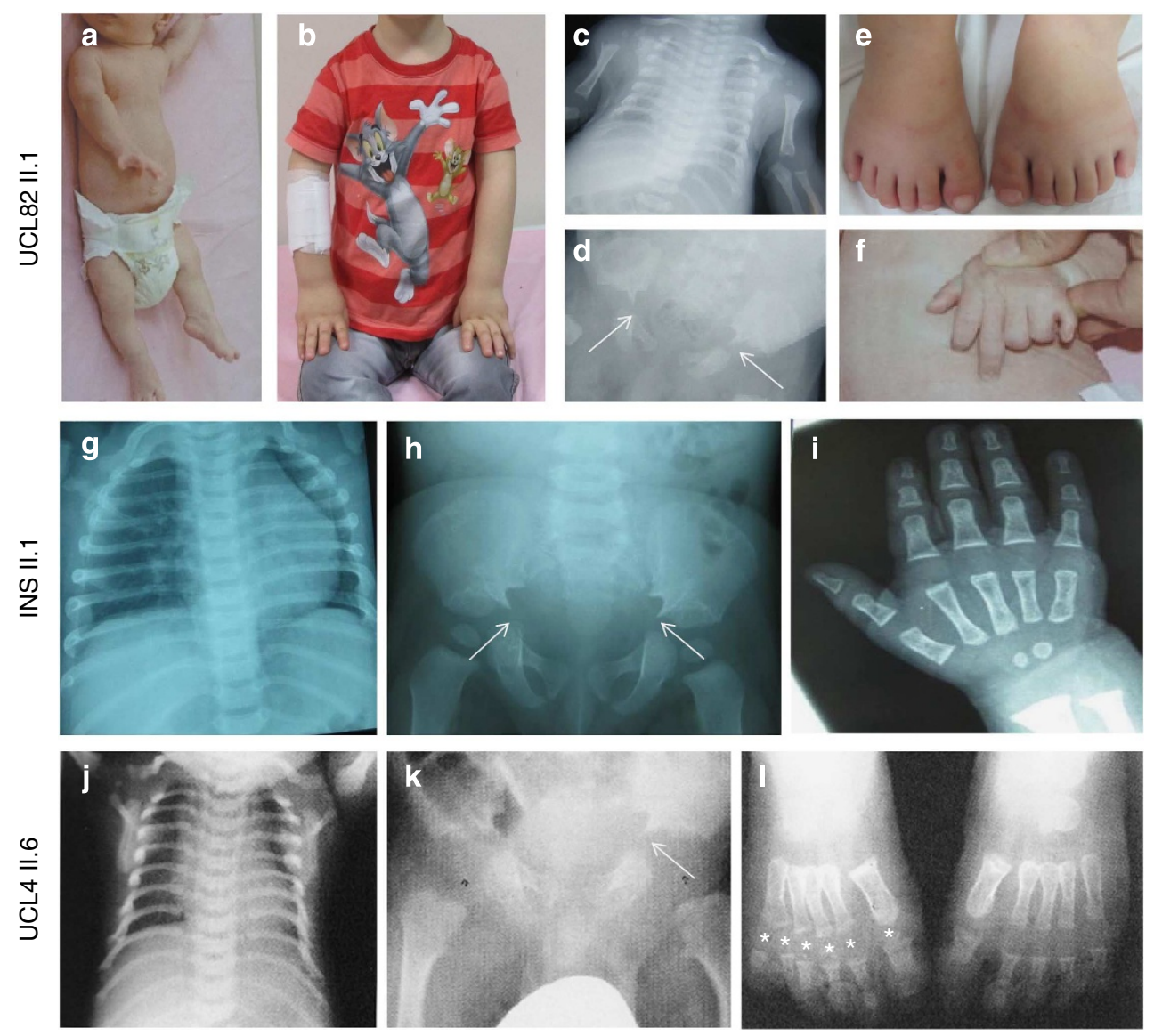

Figure 2 | Clinical features of subjects with TCTEX1D2 mutations. Affected individuals presented with narrow thorax due to shortened ribs (a-c, UCL82 II.1; $\mathbf{g}$, INS family II.1; j, UCL4 II.6), typical pelvis configuration showing trident acetabulum with spurs (arrows) (d, UCL82 II.1; h, INS family II.1; $\mathbf{k}$, UCL4 II.6), polydactyly (e,f, UCL82 II.1; I, UCL4 II.6), shortened extremities (a,b, UCL82 II.1) and brachydactyly (i, INS family II.1, asterisks indicate toes). UCL82 is shown at 38 days in $\mathbf{a}, \mathbf{c}, \mathbf{d}$ and $\mathbf{f}$ ) and $5.5 \mathrm{yrs}$ in $\mathbf{b}$ and $\mathbf{e})$.

generated by transforming an insertional mutant defective for D1bIC2 (dic5-1; Hou et al., manuscript in preparation) with C-terminal HA-tagged D1bIC2. dic5-1 cells have a severe flagellar assembly defect that is fully rescued by HA-tagged DibIC2 (Supplementary Fig. 7), indicating its functional incorporation into IFT dynein. D1bIC2-HA has a normal distribution in the cell, is expressed in flagella at approximately normal levels and sediments normally as part of a complex when the flagellar membrane-plus-matrix is fractionated on sucrose gradients (Supplementary Fig. 7). Therefore, D1bIC2-HA behaves like wildtype D1bIC2 at all levels examined.

We used anti-HA antibody-conjugated beads to immunoprecipitate D1bIC2-HA protein from the membrane-plus-matrix fraction of isolated steady-state (that is, non-assembling) flagella of the D1bIC2-HA strain. SDS-polyacrylamide gel electrophoresis (SDS-PAGE) detected bands at $\sim M_{\mathrm{r}} 90,000$ and 15,000 in the D1bIC2-HA immunoprecipitate that were absent from the wild-type control (Fig. 5a). Mass spectrometry of excised bands identified several proteins specific for the D1bIC2-HA sample, including D1bIC1 (homologue of H.s. WDR60), Tctex2b, Tctex1 (homologous to both H.s. DYNLT1 and DYNLT3) and LC8 (homologous to both H.s. DYNLL1 and DYNLL2). LC7a (homologue of H.s. DYNLRB1 and DYNLRB2) was identified by nine peptides in the D1bIC2-HA sample and two peptides in the wild-type control. Western blotting confirmed that DlbIC1, Tctex2b, Tctex1 and LC8 were specific for the D1bIC2-HA sample (Fig. 5b-e), and also identified LC7b (also a homologue of
H.s. DYNLRB1 and DYNLRB2) whose peptides were not detected in the immunoprecipitate because it migrated below the excised slice (Fig. 5c). Moreover, all detectable D1bIC1, Tctex2b and Tctexl were co-immunoprecipitated from the fraction (Fig. 5b-d). About one-half of the LC7b, about one-quarter of the DHC1b, D1bLIC and LC8, and trace amounts of FLA10 (a kinesin-2 heavy chain) and several IFT-particle proteins also were co-immunoprecipitated with D1bIC2 (Fig. 5b-e).

Tctex $2 \mathrm{~b}$ and Tctex 1 have not previously been shown to be associated with IFT dynein in Chlamydomonas, but they are known subunits of the axonemal inner arm dynein I1/f, which is involved in flagellar motility ${ }^{35}$. Dynein $\mathrm{I} 1 / \mathrm{f}$ is present at very low levels in the membrane-plus-matrix fraction of steady-state flagella ${ }^{36}$. Nevertheless, to be sure that the presence of Tctex $2 b$ and Tctex1 in the D1bIC2-HA immunoprecipitate was not due to contamination by dynein I1/f, we probed blots of the unbound and bound membrane-plus-matrix with an antibody to IC140, an $\mathrm{I} 1 / \mathrm{f}$ intermediate chain ${ }^{37}$, and found no IC140 in either the unbound or bound fractions of either the wild-type or mutant extracts. We also probed for $\mathrm{p} 28$, a subunit common to several other inner arm dyneins and previously found in the membraneplus-matrix fraction of steady-state flagella ${ }^{36}$. We readily detected p28 in the unbound fractions, but not in the bound fractions, indicating that the bound fractions were not contaminated by these other inner arm dyneins (Fig. 5e). We conclude that Tctex $2 \mathrm{~b}$ and Tctex 1 are novel components of Chlamydomonas IFT dynein. These results also show that the light chains Tctex $2 \mathrm{~b}$ 
Table 1 | Summary of clinical findings in individuals with TCTEX1D2 mutations.

\begin{tabular}{|c|c|c|c|c|c|c|c|c|c|c|}
\hline Family & Ethnicity & $\begin{array}{l}\text { Consan- } \\
\text { guineous }\end{array}$ & Patient & Mutation & $\begin{array}{l}\text { Clinical } \\
\text { diagnosis }\end{array}$ & Thorax & Polydactyly & $\begin{array}{l}\text { Other } \\
\text { skeletal } \\
\text { features }\end{array}$ & $\begin{array}{l}\text { Renal, } \\
\text { liver or } \\
\text { eye } \\
\text { phenotype }\end{array}$ & $\begin{array}{l}\text { Other } \\
\text { remarks }\end{array}$ \\
\hline & \multirow{2}{*}{$\begin{array}{c}\text { UAE } \\
\text { (Yemeni) }\end{array}$} & \multirow{2}{*}{ Yes } & 11.9 & $\begin{array}{l}\text { Homozygous } \\
\text { deletion of } \\
\text { exon } 1+2\end{array}$ & JATD & $\begin{array}{l}\text { Short } \\
\text { horizontal } \\
\text { ribs, narrow } \\
\text { thorax }\end{array}$ & $\begin{array}{l}\text { Hand and } \\
\text { foot }\end{array}$ & $\begin{array}{l}\text { Trident } \\
\text { acetabulum } \\
\text { with spurs }\end{array}$ & No & $\begin{array}{l}\text { Died aged } 2 \text { months } \\
\text { due to respiratory } \\
\text { failure }\end{array}$ \\
\hline & & & 11.5 & $\begin{array}{l}\text { Homozygous } \\
\text { deletion of } \\
\text { exon } 1+2\end{array}$ & NA & $\begin{array}{l}\text { Normal as a } \\
\text { young adult. } \\
\text { Pectus } \\
\text { carinatum } \\
\text { reported as a } \\
\text { child }\end{array}$ & No & $\begin{array}{l}\text { Not evident as a } \\
\text { young adult } \\
\text { (only mild } \\
\text { brachydactyly } \\
\text { and limb } \\
\text { shortening) }\end{array}$ & No & $\begin{array}{l}\text { Height <3.p. } \\
\text { No X-ray } \\
\text { documentation } \\
\text { during childhood } \\
\text { performed }\end{array}$ \\
\hline UCL82 & Turkish & Yes & II.1 & $\begin{array}{l}\text { Homozygous } \\
\text { splice site } \\
\text { mutation } \\
\text { c. } 113+2 \mathrm{C}>\mathrm{G}\end{array}$ & JATD & $\begin{array}{l}\text { Short } \\
\text { horizontal } \\
\text { ribs, narrow } \\
\text { thorax }\end{array}$ & $\begin{array}{l}\text { Hand and } \\
\text { foot }\end{array}$ & $\begin{array}{l}\text { Trident } \\
\text { acetabulum with } \\
\text { spurs, } \\
\text { brachydactyly }\end{array}$ & No & $\begin{array}{l}\text { Height }<3 . p \text {. } \\
\text { Alive aged } 5.5 \text { years }\end{array}$ \\
\hline
\end{tabular}

JATD, Jeune asphyxiating thoracic dystrophy; NA, not applicable; $p$, percentile.

and Tctex1, and intermediate chain D1bIC1 are more closely associated with D1bIC2 than with the heavy and lightintermediate chains DHC1b and D1bLIC. It is therefore likely that D1bIC2, D1bIC1, Tctex1, Tctex2b and LC7b together with LC8 form a discrete intermediate chain/light-chain complex within IFT dynein.

Tctex $2 b$ is involved in retrograde IFT. With this evidence that Tctex $2 b$ is a subunit of IFT dynein, we investigated whether loss of Tctex $2 b$ affects retrograde IFT. We re-examined a Chlamydomonas Tctex $2 \mathrm{~b}$ null strain (here termed tctex $2 b)^{35}$ previously shown to swim slower than wild-type cells owing to a defect in inner arm dynein I1/f, but not examined for defects in retrograde IFT. The tctex $2 b$ mutant has normal length flagella at steady state, but its rate of flagellar assembly is about one-third less than that of wild type (Fig. 6a). Differential interference contrast (DIC) microscopy revealed that anterograde IFT velocity is normal, and anterograde IFT frequency only slightly reduced (Fig. 6b,c). However, retrograde IFT velocity is $\sim 50 \%$ reduced, and retrograde IFT frequency is $<25 \%$ that of wild type (Fig. $6 \mathrm{~b}, \mathrm{c}$ ).
Therefore, loss of Tctex $2 b$ has a severe effect specifically on retrograde IFT, indicating that $\mathrm{Tctex} 2 \mathrm{~b}$ is required for normal retrograde IFT.

IFT dynein is destabilized in the absence of Tctex $2 b$. To better understand why retrograde IFT frequency and velocity are reduced in the Chlamydomonas tctex $2 b$ null mutant, we compared IFT dynein levels in wild-type and tctex $2 b$ whole-cell lysates by western blotting (Fig. 6d). DHC1b, D1bIC2 and D1bLIC were each reduced by $\sim 40-60 \%$ in the mutant cells relative to wild type. These results strongly suggest that IFT dynein is destabilized in the absence of Tctex $2 b$, with resulting degradation of the protein complex. In isolated tctex $2 b$ flagella, levels of DHC1b, D1bIC2 and D1bLIC were reduced even further (Fig. 6e) to only $\sim 15-25 \%$ that of wild type. This likely accounts for the reduced frequency of retrograde IFT in the mutant.

Western blotting also revealed that the mutant whole cells had normal levels of IFT-particle proteins (Fig. 6d), but the mutant flagella had increased amounts of all IFT complex A and B 

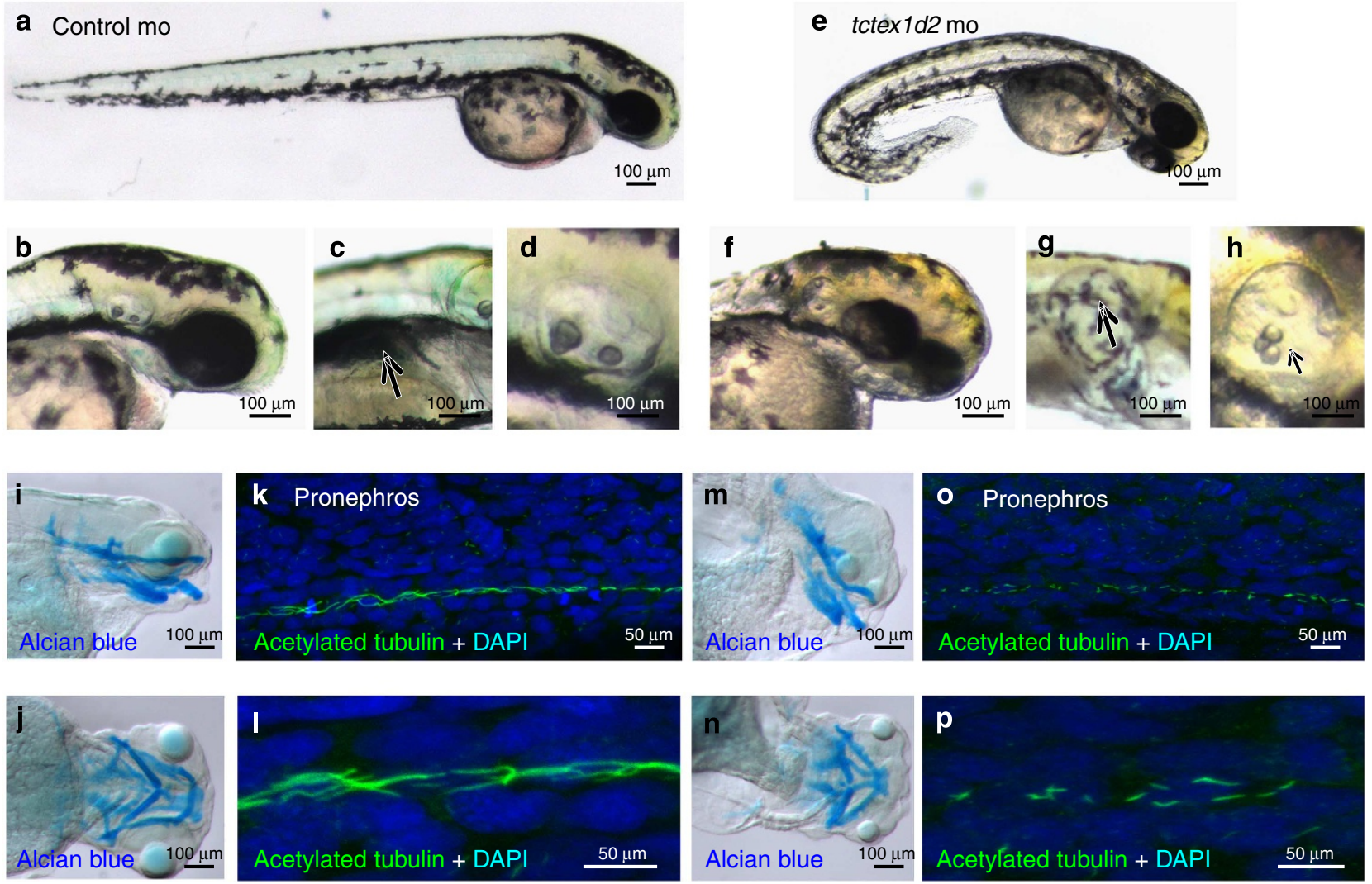

Figure 3 | Knockdown of tctex1d2 in zebrafish leads to a typical ciliopathy phenotype. Whole-mount light microscopy showing control morpholino (mo)-injected embryos (a-d) and tctex1d2 morphants at 4 days post fertilization (e-h). Compared with controls, knockdown of tctex1d2 results in ventrally curved body axis $(\mathbf{a}, \mathbf{e})$, small eyes $(\mathbf{b}, \mathbf{f})$, pronephric cysts $(\mathbf{c}, \mathbf{g})$ and otolith defects $(\mathbf{d}, \mathbf{h})$. Alcian blue staining of cartilage identifies craniofacial cartilage defects in tctexd2 morphants ( $\mathbf{m}, \mathbf{n})$ compared with controls $(\mathbf{i}, \mathbf{j})$. Immunofluorescence analysis after staining of cilia at 24 h.p.f. with anti-acetylated tubulin antibody reveals shorter cilia in the pronephric duct of tctex1d2 morphants (o, magnified in $\mathbf{p}$ ) compared with control embryos (k, magnified in $\mathbf{I})$; however, this difference was no longer evident at 48 h.p.f. (data not shown). Scale bars, $100 \mu \mathrm{m}(\mathbf{a}-\mathbf{j}, \mathbf{m}, \mathbf{n})$ or $50 \mu \mathrm{m}(\mathbf{k}, \mathbf{l}, \mathbf{o}, \mathbf{p})$.

proteins examined (Fig. 6e). This is in agreement with the IFT88 accumulation in fibroblast cilia from affected individual UCL82 II.1 (Fig. 4); both indicate a retrograde IFT defect. If nearly normal amounts of IFT complexes A and B (apparently assembled into larger IFT trains; Fig. 6b) are being moved by fewer dynein motors, it could also explain why IFT velocity is reduced in the mutant flagella. In addition, since IFT dynein is reduced much more in the mutant flagella than in the cell body, the IFT dynein lacking Tctex $2 b$ is likely to be imported less efficiently into flagella than is the complete IFT dynein.

TCTEX1D2 is a component of human IFT dynein. To confirm that TCTEX1D2 is part of human IFT dynein, we performed affinity proteomics after expressing SF-TAP (Streptavidin/FLAG Tandem Affinity Purification)-tagged TCTEX1D2 in HEK293T cells; cells expressing SF-TAP-RAF1 served as a control. We were able to identify interactions of TCTEX1D2 with IFT dynein intermediate chains WDR34 and WDR60, and light chains DYNLT1, DYNLT3 and DYNLRB1 (Supplementary Table 7). No peptides from cytoplasmic dynein 1 intermediate chains were detected, ruling out possible contamination by that dynein. An interaction between TCTEX1D2 and WDR60 was verified after overexpression of Flag-tagged TCTEX1D2 and GFP-tagged WDR60 in HEK293T cells (Supplementary Fig. 8). Identification of TCTEX1D2, DYNLT1, DYNLT3 and DYNLRB1 as part of the human IFT dynein is consistent with our finding that the homologous Tctex $2 b$, Tctex 1 and LC7b are part of IFT dynein in
Chlamydomonas, and provides independent evidence that IFT dynein contains several different light chains. A model of IFT dynein based on these findings is shown in Fig. 7.

\section{Discussion}

Here we report that TCTEX1D2/Tctex2b is a light chain of IFT dynein in humans and Chlamydomonas, and show for the first time that it is essential for IFT dynein stability and normal retrograde (tip-to-base) IFT. In zebrafish, tctex $1 d 2$ gene silencing suggests a role conserved across vertebrates. We show that biallelic loss-of-function alleles in this gene in humans cause JATD. In all affected individuals, we identified biallelic TCTEX1D2 null alleles, which is in striking contrast to JATD and SRPS individuals carrying mutations in other IFT dynein genes such as DYNC2H1 (refs 15,16,34,38,39), WDR34 (refs $12,13)$, WDR60 (ref. 14) or in genes encoding IFT-A ${ }^{21}$ and IFT$\mathrm{B}^{18,20}$ components. These individuals usually carry at least one missense allele with inheritance restricted to two such hypomorphic alleles or one hypomorphic allele in combination with a null mutation ${ }^{13,17,39}$. Biallelic null alleles also cause early embryonic lethality in mouse IFT dynein knockouts ${ }^{25,26,40-42}$, and complete loss of IFT dynein subunits was thus to date considered incompatible with embryonic development beyond midgestation. Our data suggest that TCTEX1D2 is an exception to this dogma, since all but one child carrying TCTEX1D2 null mutations survived beyond infancy.

The high survival rate could reflect a milder thorax phenotype than in individuals with mutations in other IFT dynein genes 

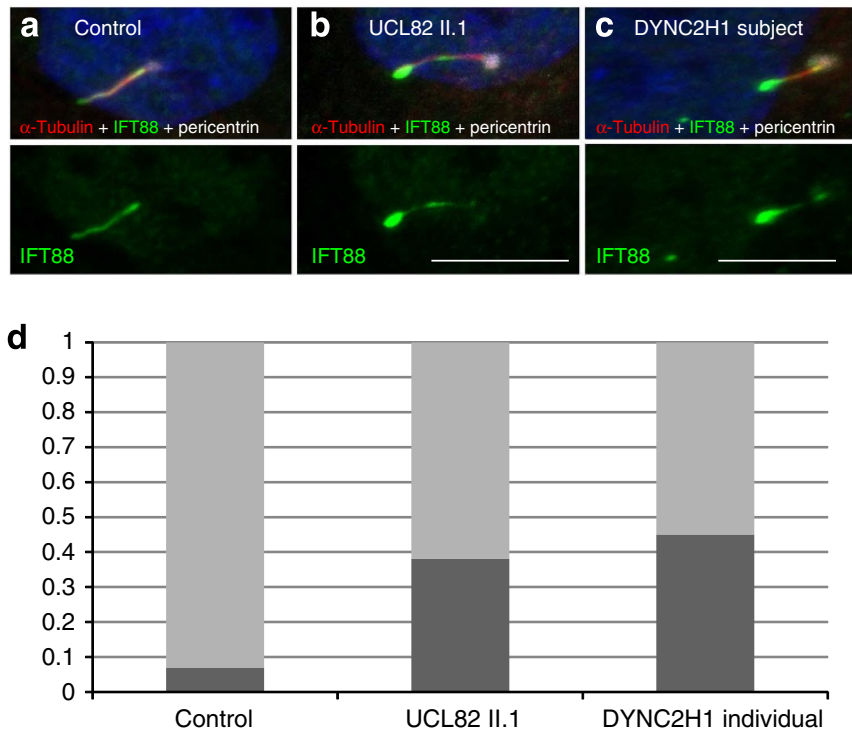

- IFT88 accumulation at the ciliary tip Normal IFT88 distribution
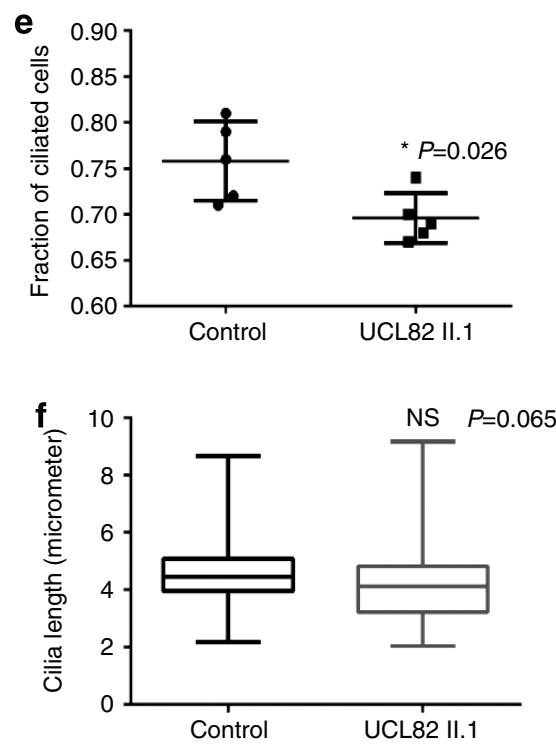

Figure 4 | Loss of human TCTEX1D2 results in retrograde IFT defects. Immunofluorescence analysis using confocal microscopy revealed an accumulation of IFT88 at the ciliary tips in skin fibroblasts from individual UCL82 II.1 compared with a control (a,b). The accumulation is comparable to that previously reported in fibroblast cilia from an individual with JATD caused by biallelic variants in DYNC2H1 (ref. 34) (c). IFT88 staining is shown in green, anti-acetylated tubulin antibody (red) was used for visualization of the ciliary axoneme, anti-pericentrin antibody (white) marks the ciliary base; the IFT88 labeling also is shown separately in the lower panels. Scale bars, $5 \mu \mathrm{m}$. (d) Fraction of cells with IFT88 accumulation at the ciliary tip, 100 cells analysed for each condition. (e) The percentage of ciliated cells in the fibroblast sample from individual UCL82 II.1 compared with control fibroblasts as assessed by counting the number of cilia stained with anti-acetylated tubulin antibody in relation to nuclei stained with DAPI (4,6-diamidino-2-phenylindole, blue in a-c) in 10 random visual fields in five independent experiments each, revealing a very mild reduction in ciliation for the TCTEX1D2-deficient cells. One hundred cells were counted per experiment, represented by a single point per experiment. (f) No difference in cilia length between UCL82 II. 1 and control fibroblasts was visualized using anti-acetylated tubulin antibody, 100 cells analysed for each condition. Statistical significance in $\mathbf{e}$ and $\mathbf{f}$ was measured using the Student's $t$-test, asterisk indicates $P$ value $<0.05$.

such as DYNC2H1, WDR34 and WDR60 (refs 12-15,34). Similarly to individuals with mutations in the IFT dynein heavy-chain gene DYNC2H1 (refs 15,34), individuals with TCTEX1D2 mutations did not show signs of extraskeletal disease; however, these phenotypes could still emerge with age. Two siblings (UCL4 II.1 and II.5) carrying homozygous TCTEX1D2 mutations did not show overt signs of JATD, suggesting disease non-penetrance. Both are now young adults, and it is possible that early JATD radiological features (handlebar clavicles, trident acetabulum with spurs and cone-shaped epiphyses) were missed because a full X-ray exam was only performed in adulthood after we detected the unusual penetrance pattern. However, in contrast to their three affected siblings, they also do not exhibit polydactyly or shortened ribs. Although both are short statured with mildly short digits and lower limbs, more specific radiological signs of JATD/SRPS were not found.

Together, these clinical findings suggest a less essential role of TCTEX1D2 in ciliary transport mechanisms compared with other proteins mutated in JATD and SRPS. Cilia of human fibroblasts with TCTEX1D2 mutations and tctex1d2 zebrafish morphants lack gross structural defects, indicating a potentially non-essential role in vertebrate ciliogenesis. Similarly, flagella of the Chlamydomonas tctex $2 b$ null mutant are normal length at steady state, whereas null mutants of IFT dynein subunits DHC1b or D1bLIC have a severe short flagellar phenotype ${ }^{43-45}$.

Our studies in Chlamydomonas provide insight into the molecular mechanism underlying the milder phenotype. Loss of Chlamydomonas Tctex $2 \mathrm{~b}$ causes IFT dynein instability and a reduced amount of IFT dynein in flagella; however, surprisingly, the residual IFT dynein lacking Tctex $2 b$ retains enough functionality to maintain full-length flagella. Thus, it appears that a key role of Tctex $2 b$ is to stabilize IFT dynein. The lower level of IFT dynein is accompanied by reduced retrograde IFT velocity and frequency, causing an accumulation of IFT-particle proteins both in Chlamydomonas flagella and skin fibroblast cilia from affected individual UCL82 II.1. That Chlamydomonas cells tolerate some reduction of retrograde IFT is in agreement with observations that the $d h c 1 b$ temperature-sensitive mutants dhc1b-3 and fla24 have reduced amounts of IFT dynein and reduced retrograde IFT at permissive temperature, yet still assemble normal length flagella ${ }^{46-48}$. In the case of the tctex $2 b$ mutant, flagella are formed more slowly than in wild-type cells, probably reflecting slower recycling of IFT components.

The slower flagellar assembly in the Chlamydomonas tctex $2 b$ mutant, if recapitulated in vertebrates, might explain our observation that tctex $1 d 2$ morphant zebrafish embryos appeared to have short cilia at 24 h.p.f. but normal length cilia at 48 h.p.f. Interestingly, this finding, and the normal length cilia and flagella found in patient UCL82 II.1 fibroblasts and the Chlamydomonas tctex $2 b$ mutant, contrasts with a recent report that short interfering RNA-mediated depletion of TCTEX1D2 in human telomerase-immortalized retinal pigment epithelial (hTERTRPE1) cells resulted in longer cilia ${ }^{49}$. However, cilia length changes, ranging from shorter, normal length or longer than normal ${ }^{12,18,34}$, have been reported in cells from JATD patients with a number of gene defects. Furthermore, WDR34 mutant patient fibroblasts have shortened cilia ${ }^{12}$, whereas WDR34 short interfering RNA knockdown in hTERT-RPE1 results in longer cilia $^{49}$. Vertebrate cilia length changes arising from retrograde IFT perturbations may thus be cell type specific or subject to experimental variability. These changes are not necessarily predictable, and therefore do not seem to provide a reliable 
a
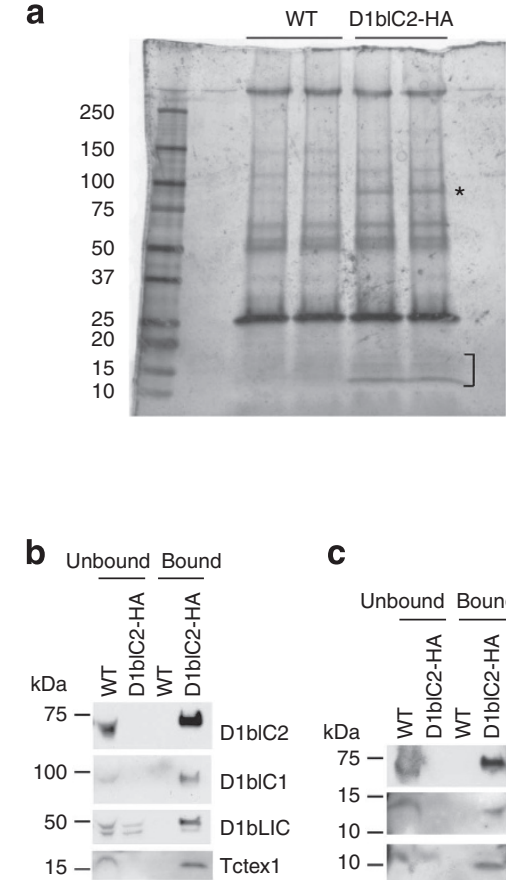

c
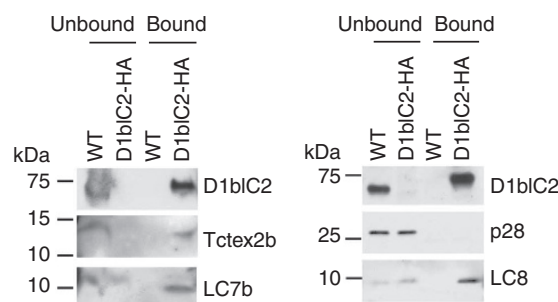

Figure 5 | Tctex2b and Tctex1 are in an IFT dynein intermediate chain/light-chain subcomplex with D1bIC2 and D1bIC1. (a) Flagellar membrane-plus-matrix fractions from wild-type (WT) cells or cells expressing D1bIC2-HA were incubated with anti-HA antibody-conjugated beads. The proteins pulled down by the beads were separated by SDS-PAGE and silver stained. One band (marked *) between 75 and $100 \mathrm{kDa}$ and several bands around $15 \mathrm{kDa}$ (marked ]) are specific for the D1bIC2-HA sample. (b-d) Western blots confirming that Tctex2b, Tctex1 and D1bIC1 are specifically co-precipitated with D1bIC2-HA. The unbound and bound samples were probed with the indicated antibodies. In each experiment, all of the D1bIC2-HA was immunoprecipitated from the D1bIC2-HA sample; all of the D1bIC1 (b,d), Tctex1 (b) and Tctex2b (c) was co-precipitated from the D1bIC2-HA sample. None of these proteins were pulled down from the WT control. Some but not all of the DHC1b (d), D1bLIC (b,d), LC7b (c) and LC8 (e) was co-precipitated from the D1bIC2-HA samples; (d) also shows that only very small amounts of the IFT-particle proteins or FLA10 were co-precipitated with D1bIC2-HA.

(e) Similar western blot showing that p28 was not co-precipitated with D1bIC2-HA. In $\mathbf{b}$, d and $\mathbf{e}$, the ratio of unbound: bound protein loaded was $1: 4$; in c, the ratio was 1:2. All antibodies used for Chlamydomonas protein analysis are listed in Supplementary Table 6.

measure, compared with the direct IFT measurements reported here that include immunofluorescence imaging of altered localization of IFT cargos and DIC imaging of IFT velocity. Although no difference in cilia length was observed in skin fibroblasts of JATD compared with control individuals in our study, similar to the Chlamydomonas Tctex $2 b$ mutant, any delay in cilia assembly could have severe consequences during development if full-length cilia are required for proper functioning of the hedgehog or other vertebrate ciliary signalling pathways soon after onset of cilia formation. Since retrograde IFT is critical for hedgehog signalling ${ }^{11,50}$, impaired retrograde IFT even in full-length cilia could be expected to affect skeletal development, as seen in the TCTEX1D2-mutated JATD individuals.

The Chlamydomonas Tctex $2 \mathrm{~b}$ mutant swims $\sim 30 \%$ slower than wild-type cells owing to defects in axonemal dyneins ${ }^{35}$. However, a ciliary motility defect is not the cause of JATD, because primary ciliary dyskinesia (PCD) patients with ciliary motility defects do not have JATD symptoms, and multiciliated cells bearing motile cilia are not present in the tissues affected in JATD nor in their progenitor cells ${ }^{1}$. JATD is caused by defects in primary cilia; primary cilia lack axonemal dyneins.

Proteomic analysis in Chlamydomonas and human cells confirmed Tctex2b/TCTEX1D2 as a component of IFT dynein along with intermediate chains D1bIC2/WDR34 and D1bIC1/ WDR60, and a number of light chains. Immunoprecipitation of Chlamydomonas IFT dynein not only identified Tctex $2 \mathrm{~b}$ but also Tctex1 and LC7b as novel subunits of IFT dynein, indicating that these light chains together with LC8, D1bIC2 and D1bIC1 likely form a light-chain/intermediate chain complex within IFT dynein, which also may contain subunits not detected by our methods. Proteomics analysis of HEK293T proteins co-immunoprecipitated with SF-TAP-tagged TCTEX1D2 likewise identified DYNLT1 and DYNLT3 (human Tctex1), and additional light chains in the LC8 and roadblock families (DYNLL2 = LC8-type 2 and DYNLRB1 = DNLC2A). The light-chain pairs DYNLL1/ DYNLL2 and DYNLRB1/DYNLRB2 have a high sequence identity of $93 \%$ and $77 \%$ respectively. This approach did not identify any peptides specific for DYNLL1 or DYNLRB2, but one peptide each was identified for DYNLL1/DYNLL2 and DYNLRB1/DYNLRB2, which could have originated from either of the two homologous proteins. Furthermore DYNLL1 was previously confirmed as a light chain of human IFT dynein ${ }^{13}$. Therefore, we propose that these subunits are components of an ancient, highly conserved intermediate chain/light-chain complex within IFT dynein (Fig. 7). As such, these light chains are strong candidates for harbouring novel mutations causing JATD.

Dynein complexes fall into two general types based on whether they contain single or multiple heavy-chain motor units ${ }^{51}$. Those with multiple motors all associate with a core group of accessory proteins including WD-repeat intermediate chains and light chains in the Dynll/LC8, Dynlt/Tctex1 and Dynlrb/LC7 classes (axonemal outer dynein arms and inner arm I1/f also include proteins related to TCTEX1D2/Tctex2, which form a distinct subfamily within the Dynlt/Tctex1 group ${ }^{51}$ ). Our demonstration that the IFT dynein includes a member of this subfamily suggests that the presence of TCTEX1D2 is also a conserved defining feature of these multimotor dyneins, and raises the possibility that canonical cytoplasmic dynein 1 might also associate with members of this light chain type under certain circumstances ${ }^{52}$.

The possible disease non-penetrance in family UCL4 is of clinical interest. Variable phenotypic severity is well documented for JATD/SRPS even within families. However, never before to the extent that individuals can appear clinically unaffected but carry biallelic loss-of-function variants that cause documented JATD/SRPS in other family members. Functional redundancy of TCTEX1D2 with other proteins could explain this, if, for example, other dynein light chains might in certain conditions be able to compensate for its loss. Mice carrying Dynll1 mutations showed possible functional redundancy of IFT dynein light chains, whereby DYNLL2 may be able to function in place of DYNLL1 (ref. 53). We looked at single-nucleotide polymorphism haplotypes across dynein light-chain genes DYNLL1, DYNLL2 and TCTEX1 in individuals UCL4 II.1 and II.5 who carry the TCTEX1D2 deletion but appear clinically unaffected. Both have different haplotypes across TCTEX1 compared with their siblings II.8 and II.9 who display a classical JATD phenotype, but whether protective alleles could exist in compensatory genes is not clear (Supplementary Fig. 9).

Alternatively, TCTEX1D2 loss could be attenuated by individual-to-individual variation in the proteins with which TCTEX1D2 normally interacts, or in other proteins involved in IFT dynein stabilization or degradation. Modifier alleles have been debated as the underlying cause of the phenotypic variability 


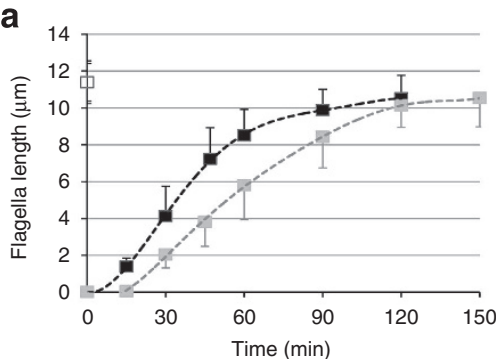

- - A54-e18 --ter-tex2b $\square$ Before deflagellation

b

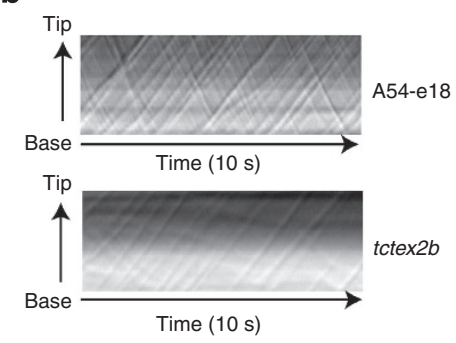

$\mathbf{C}_{4.5}$

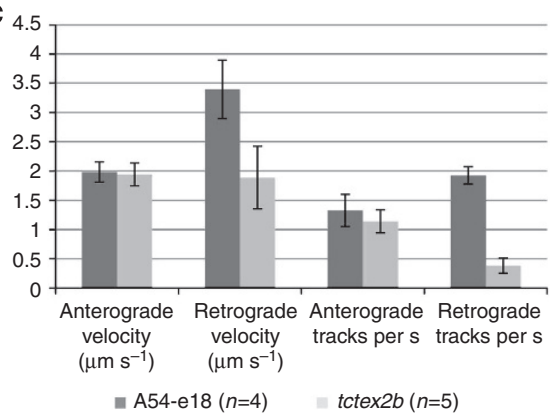

e

d

Whole cell

A54-e18 tctex2b

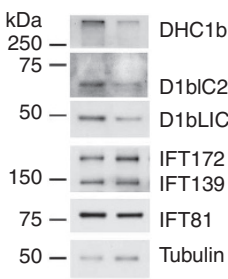

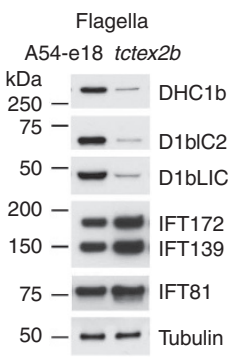

Figure $\mathbf{6}$ | Loss of Tctex $\mathbf{2 b}$ causes IFT dynein instability and a retrograde IFT defect in Chlamydomonas. (a) The tctex $2 b$ mutant has defects in flagella regeneration. The tctex $2 b$ null mutant and A54-e18 (the wild-type strain from which tctex $2 b$ was derived) were deflagellated and then allowed to regrow their flagella. Flagella lengths were measured before deflagellation and at time points after deflagellation. The two strains had identical flagellar lengths $(11.4 \mu \mathrm{m})$ before deflagellation. For each time point, one flagellum from each of 50 cells was measured; error bars are s.d. (b) The tctex $2 b$ mutant is defective in retrograde IFT. IFT was recorded in wild-type (A54-e18) and tctex2b flagella by DIC microscopy, and kymograms generated from the video recordings. Tracks with positive slopes represent IFT particles moving anterogradely, and tracks with negative slopes represent particles moving retrogradely. Compared with wild type, few retrograde tracks are visible in the tctex $2 b$ kymogram, and these had a much reduced slope. Retrograde particles had a larger apparent size in mutants; similar findings were reported for a temperature-sensitive $d h c 1 b$ mutant ${ }^{47}$. (c) Quantitative analysis of IFT in wild type (A54-e18) and tctex2b. In tctex2b, anterograde IFT velocity is about the same as in wild type, while anterograde frequency is only slightly reduced, but both retrograde IFT velocity and frequency are greatly reduced. $n$, number of flagella analysed. Error bars show s.d. (d) Western blot showing reduced IFT dynein subunits in tctex $2 b$ whole-cell lysates. Wild-type (A54-e18) and tctex $2 b$ whole-cell lysates were probed with antibodies to IFT dynein subunits and IFT-particle proteins. DHC1b, D1bIC2 and D1bLIC are reduced in tctex $2 b$ whole-cell lysate. No significant changes were detected for IFT proteins. The same samples were probed for tubulin as loading control. (e) IFT dynein is greatly reduced in tctex $2 b$ flagella. Wild-type (A54-e18) and tctex2b flagella were probed with antibodies to IFT dynein subunits and IFT-particle proteins. IFT dynein subunits DHC1b, D1bIC2 and D1bLIC are greatly reduced in tctex $2 b$ flagella. IFT-A protein IFT139 and IFT-B proteins IFT172 and IFT81 are increased in tctex2b flagella, consistent with a retrograde IFT defect. The same samples were probed for tubulin as loading control.

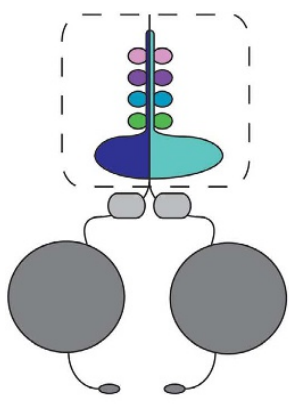

Dynein $2(\mathrm{Hs}) /$ Dynein $1 \mathrm{~b}(\mathrm{Cr})$

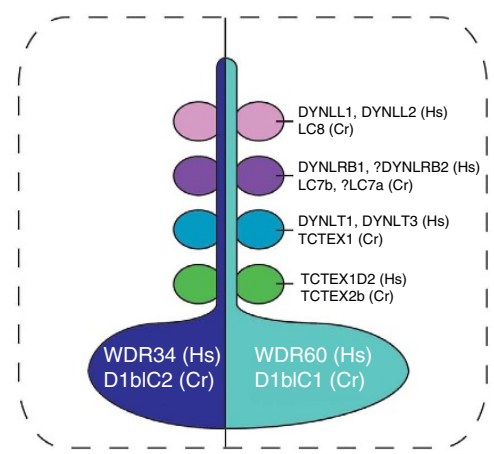

Figure 7 | Proposed model of IFT dynein composition in Homo sapiens (Hs) and $\mathbf{C}$. reinhardtii (Cr). Left, IFT dynein (dynein 2/1b) is composed of dynein heavy chains (DYNC2H1(Hs)/DHC1b(Cr); shown in dark grey), dynein light-intermediate chains (DYNC2LI1(Hs)/D1bLIC(Cr); shown in light grey) and different dynein intermediate and light chains (coloured, shown in detail on the right). Right, dynein intermediate chains (WDR34(Hs)/D1bIC2(Cr) and WDR60(Hs)/D1bIC1(Cr)) interact with different dynein light-chain subtypes, including TCTEX1D2(Hs)/ TCTEX2b(Cr). Question marks indicate LC7a and DYNLRB2 as unconfirmed components suggested from our Chlamydomonas and human results. in ciliary chondrodysplasias ${ }^{13,23,34}$ and other ciliopathies such as Bardet-Biedl, Joubert and Meckel syndromes; however, this has remained a challenge to prove in human subjects ${ }^{54,55}$. Variable penetrance in autosomal recessive conditions is still quite unusual, one example being a common CFTR variant that causes either disease or a normal phenotype depending on its genetic context ${ }^{56}$.

It may be relevant that partial suppression of mutations in genes encoding IFT-particle proteins has been observed in Chlamydomonas and Tetrahymena ${ }^{57-59}$; this occurs in association with stress, suggesting possible involvement of a chaperone in stabilizing the incomplete IFT particle. Given that a key role of Tctex $2 b$ is to stabilize IFT dynein, the presence or absence of another protein such as a chaperone that helps stabilize IFT dynein could determine whether or not a developmental defect is observed in the absence of Tctex $2 b$. Interestingly, peptides derived from chaperones were abundant in our proteomic analysis of TAP-tagged TCTEX1D2 from HEK293T cells.

In summary, we have identified mutations associated with a complete loss of TCTEX1D2 causing Jeune syndrome. TCTEX1D2 mutations are a rare cause of disease affecting $<5 \%$ of cases in this study (three affected families identified after screening of $>300$ individuals with JATD/SRPS). There are 
important implications for genetic counselling since individuals carrying TCTEX1D2 mutations appear to have a higher survival rate than individuals affected by mutations in other IFT dynein components, and lack extraskeletal symptoms. In humans, fish and Chlamydomonas, TCTEX1D2 mutations do not confer changes in gross ciliary structure, but do impair the highly conserved retrograde IFT machinery. Proteomic analysis provides evidence that TCTEX1D2/Tctex2b is a component of an intermediate chain/light-chain subcomplex within IFT dynein. In Chlamydomonas, the retrograde IFT defect is associated with instability of IFT dynein; motor stability may be the major role of Tctex $2 b$ since the residual IFT dynein missing Tctex $2 b$ retains some functionality. Our findings that IFT is impaired to a lesser extent in TCTEX1D2/Tctex2b null cells than in cells carrying null mutations in other IFT dynein components could explain, in part, the apparently incomplete penetrance underlying TCTEX1D2 disease. This potentially has future clinical therapeutic implications, for example, if modulation of other components affecting the IFT dynein system could be harnessed to attenuate the effects of a lack of TCTEX1D2 function.

\section{Methods}

Patients. Inclusion criteria were the clinical diagnosis of JATD based on clinical and radiological findings including short ribs with small/narrow thorax and small ilia with acetabular spurs, handlebar clavicles and brachydactyly. All samples were obtained with approval of the UCL-ICH/Great Ormond Street Hospital Research Ethics Committee (08/H0713/82), South Yorkshire Research Ethics Committee $(11 / \mathrm{H} 1310 / 1)$ and collaborating institutions with informed consent.

Human Sanger sequencing and PCR. Familial segregation in accordance with a recessive inheritance pattern was confirmed by Sanger sequencing of PCR products amplified from genomic DNA samples of all available family members (SourceBiosciences, Cambridge, UK). For family UCL4, fresh repeat samples were collected for segregation analysis, indicating no chance of sampling error. For RT-PCR, the Omniscript kit (Qiagen) was used to make cDNA from total RNA isolated from lymphocytes or fibroblasts of affected individuals and controls using Trizol-chloroform extraction. PCR and RT-PCR primers used are listed in Supplementary Table 1.

Western blotting. Uncropped images of all western blots are included in Supplementary Fig. 10.

Constructs used in mammalian cells. Mouse WDR60 and human TCTEX1D2 cDNA were obtained from the University of Queensland's SRC Microarray facility. A sequence-verified SF-TAP-TCTEX1D2 construct (BC021177.2) containing a double Streptavidin II and a single FLAG tag was kindly provided by Nicholas Katsanis (Duke University, Durham, USA). WDR60 was cloned into the GFP-N1 vector by PCR amplification, EcoRI/KpnI digestion and ligation. TCTEX1D2 cloned into a modified pCR3 vector for expression of FLAG-tagged proteins was a kind gift from Dr Jörg Heierhorst (St Vincent's Institute of Medical Research, Melbourne). The human tGFP-tagged WDR34 mammalian expression plasmid was obtained from OriGene Technologies, Inc. (TrueORF clone RG204288).

Human cell culture. Human fibroblasts obtained by skin biopsy from study subjects and HEK293T cells (European Collection of Cell Cultures) were cultured under standard conditions at $37^{\circ} \mathrm{C}$ and $5 \% \mathrm{CO}_{2}$ in DMEM-F12 Glutamax medium (Life Technologies) with $10 \%$ fetal bovine serum (Life Technologies).

Immunofluorescence in mammalian cells and zebrafish. Human fibroblasts were split onto glass coverslips and grown until confluent. For ciliogenesis experiments, cells were serum starved using cell medium without fetal bovine serum for $20 \mathrm{~h}$. For immunofluorescence, cells were then fixed in $4 \%$ paraformaldehyde (PFA) for $10 \mathrm{~min}$, washed five times with PBS, treated with $0.05 \%$ Triton X-100/PBS for $2 \mathrm{~min}$, washed five times with PBS, blocked $1 \mathrm{~h}$ using $4 \%$ BSA in PBS and then incubated with the primary antibody. These were mouse monoclonal anti-acetylated tubulin 1:1,000 (IgG2b, clone 6-11-b1, Sigma), mouse monoclonal anti-pericentrin IgG1 1:200 (mAbcam 28144, Abcam) or rabbit polyclonal anti-IFT88 1:100 (13967-1-AP, Proteintech) overnight at $4{ }^{\circ} \mathrm{C}$. Cells were then washed again five times with $\mathrm{PBS}$ and incubated with the appropriate secondary antibodies: goat anti mouseIgG1 Alexa Fluor 647, goat anti-mouse IgG2b Alexa Fluor 568 or goat anti-rabbit Alexa Fluor 488. Glass slides were then washed five times in PBS, incubated with 4,6-diamidino-2-phenylindole (Molecular Probes, Invitrogen) for $5 \mathrm{~min}$ to obtain nuclear stain, washed five times in PBS and then mounted in Vectashield (Vector Laboratories). For zebrafish immunofluorescence studies, embryos were fixed at 24 h.p.f. in $4 \%$ PFA overnight, washed five times in PBS containing $0.1 \%$ Triton X-100/PBS, incubated in $10 \%$ methanol for $30 \mathrm{~min}$ at $-20^{\circ} \mathrm{C}$, washed five times in PBS containing $0.1 \%$ Triton X-100 and blocked with 5\% BSA in PBS containing 0.1\% Triton X-100 for $1 \mathrm{~h}$. Embryos were then incubated with the primary antibody mouse monoclonal anti-acetylated tubulin 1:1,000 (IgG2b, clone 6-11-b1, Sigma) or mouse monoclonal anti-gamma tubulin 1:250 (IgG1, Sigma) overnight at $4{ }^{\circ} \mathrm{C}$, washed five times in PBS containing $0.1 \%$ Triton X-100/PBS and then incubated with the appropriate secondary antibodies: goat anti-mouse IgG1 Alexa Fluor 647 1:1,000 or goat anti-mouse IgG2b Alexa Fluor 488 1:1,000 and 4,6-diamidino-2-phenylindole 1:25,000. All imaging was performed using a Zeiss LSM710 confocal microscope.

Statistical analysis. Statistical analysis of cilia number and cilia length was performed using Student's $t$-test with statistical analysis performed using GraphPad Prism.

Chlamydomonas cells and culture conditions. Chlamydomonas reinhardtii strain 137c (nit1, nit2, $m t+$ ) from the Chlamydomonas Resource Center (University of Minnesota, St Paul, MN) was used as wild type. A54-e18 (nit1-1, ac17, sr1, mt +), which is the parent of $p f 16-\mathrm{D} 2$ (a double mutant of $p f 16$ and tctex $2 b$ ), and ' $p f 16-\mathrm{D} 2$ Resc. w/PF16,' which is pf16-D2 rescued for the PF16 gene ${ }^{35,60}$, were from Elizabeth Smith (Dartmouth College, NH). The dic5-1 insertional mutant was generated by transforming g1 cells (nit1, NIT2, $\mathrm{mt}+)^{61}$ with the 1.7 -kb chimeric aph7" gene cut by HindIII from the Hyg3 plasmid $^{62}$, followed by backcrossing of a transformant to wild-type cells. The D1bIC2-HA strain was generated by transformation of dic5-1 with a gene encoding D1bIC2 with a C-terminal $3 \times \mathrm{HA}$ tag. Cells were grown in TAP medium ${ }^{63}$ or $\mathrm{M}$ medium I ${ }^{64}$ altered to have $0.0022 \mathrm{M}$ $\mathrm{KH}_{2} \mathrm{PO}_{4}$ and $0.00171 \mathrm{M} \mathrm{K}_{2} \mathrm{HPO}_{4}$; cultures were either aerated with $5 \% \mathrm{CO}_{2}$ and $95 \%$ air or grown on 24 -well plates.

Creation of DNA constructs for Chlamydomonas transformation. The wild-type DIC5 gene was amplified by PCR from genomic DNA using primer pairs FAP1332 (5'-TGTCCCGCTGCAGAGCAATG- $3^{\prime}$ ) and FAP133-3 (5'-ACCCCG CCTCCTTGTCCTTG- $3^{\prime}$ ). The blunt ends of the PCR product were modified by A-tailing, that is, an extra A was added at the $3^{\prime}$ ends of the product strands by Taq polymerase. The modified PCR product was cloned into pGEM-T (Promega, Madison, MI). To insert an HA tag into the DIC5 gene just before the stop codon, primer pair FAP133-15 (5'-GTAGAGTGGCAGTGCCGGC-3') and FAP133-16 (5'-GCGTGAAGTTGCCGCGCA- $\left.3^{\prime}\right)$ was used to amplify the DIC5 gene and vector. The PCR product was then ligated to the $3 \times$ HA fragment excised from plasmid p $3 \times \mathrm{HA}^{65}$ by SmaI.

Chlamydomonas immunoprecipitation and mass spectrometry. Flagella membrane-plus-matrix fractions from wild-type and D1bIC2-HA cells were incubated with the anti-HA affinity matrix (Roche Diagnostics $\mathrm{GmbH}$ ) overnight. The beads were washed three times with HMEK buffer (30 mM HEPES, pH 7.4, $5 \mathrm{mM}$ $\mathrm{MgSO}_{4}, 0.5 \mathrm{mM}$ EGTA and $25 \mathrm{mM} \mathrm{KCl}$ ) plus $0.01 \% \mathrm{NP}-40$. Protease Inhibitor Cocktail for plant cell and tissue extracts (Sigma-Aldrich) was added to prevent protein degradation. SDS-PAGE sample loading buffer was used to elute the proteins from the matrix. Proteins in the bound fractions from wild-type and D1bIC2-HA samples were then separated by SDS-PAGE and stained with Silver Stain Plus (Bio-Rad). Gel regions of interest were excised and analysed by mass spectrometry at the Proteomics and Mass Spectrometry Facility, University of Massachusetts Medical School, and the Vermont Genetics Network Proteomics Facility, University of Vermont ${ }^{66,67}$.

Flagellar regeneration and analysis of IFT. For DIC imaging of steady-state flagella and the subsequent analysis of IFT, live cells were immobilized in $1 \%$ agarose and observed using an inverted microscope (Ti U; Nikon) equipped with DIC optics ${ }^{59}$. To determine the kinetics of flagellar regeneration, cells were deflagellated by the $\mathrm{pH}$-shock method ${ }^{68}$ and allowed to regrow flagella under the same conditions as before deflagellation. Aliquots of the cell suspensions were removed at various times and fixed with $1 \%$ glutaraldehyde. Images of the fixed cells were acquired with an AxioCam camera, AxioVision 3.1 software, and an Axioskop 2 plus microscope (Zeiss). Flagellar lengths were measured using ImageJ (http://rsb.info.nih.gov/ij/index.html). Images were processed using Adobe Photoshop (Adobe Systems Incorporated, San Jose, CA).

\section{References}

1. Fliegauf, M., Benzing, T. \& Omran, H. When cilia go bad: cilia defects and ciliopathies. Nat. Rev. Mol. Cell. Biol. 8, 880-893 (2007).

2. Hildebrandt, F., Benzing, T. \& Katsanis, N. Ciliopathies. N. Engl. J. Med. 364, 1533-1543 (2011).

3. Pedersen, L.B. \& Rosenbaum, J.L. Intraflagellar transport (IFT) role in ciliary assembly, resorption and signalling. Curr. Topics Dev. Biol. 85, 23-61 (2008). 
4. Patel-King, R.S., Gilberti, R.M., Hom, E.F. \& King, S.M. WD60/FAP163 is a dynein intermediate chain required for retrograde intraflagellar transport in cilia. Mol. Biol. Cell 24, 2668-2677 (2013).

5. Hom, E.F.Y. et al. A unified taxonomy for ciliary dyneins. Cytoskeleton (Hoboken NJ) 68, 555-565 (2011).

6. Pazour, G.J., Wilkerson, C.G. \& Witman, G.B. A dynein light chain is essential for the retrograde particle movement of intraflagellar transport (IFT). J. Cell Biol. 141, 979-992 (1998).

7. Rompolas, P., Pedersen, L.B., Patel-King, R.S. \& King, S.M. Chlamydomonas FAP133 is a dynein intermediate chain associated with the retrograde intraflagellar transport motor. J. Cell Sci. 120, 3653-3665 (2007).

8. Mikami, A. et al. Molecular structure of cytoplasmic dynein 2 and its distribution in neuronal and ciliated cells. J. Cell Sci. 115, 4801-4808 (2002).

9. Grissom, P.M., Vaisberg, E.A. \& McIntosh, J.R. Identification of a novel light intermediate chain (D2LIC) for mammalian cytoplasmic dynein 2. Mol. Biol. Cell 13, 817-829 (2002).

10. Pfister, K.K. et al. Genetic analysis of the cytoplasmic dynein subunit families. PLoS Genet. 2, el (2006).

11. Goetz, S.C. \& Anderson, K.V. The primary cilium: a signalling centre during vertebrate development. Nat. Rev. Genet. 11, 331-344 (2010).

12. Huber, C. et al. WDR34 mutations that cause short-rib polydactyly syndrome type III/severe asphyxiating thoracic dysplasia reveal a role for the NF-kappaB pathway in cilia. Am. J. Hum. Genet. 93, 926-931 (2013).

13. Schmidts, M. et al. Mutations in the gene encoding IFT dynein complex component WDR34 cause Jeune asphyxiating thoracic dystrophy. Am. J. Hum. Genet. 93, 932-944 (2013).

14. McInerney-Leo, A.M. et al. Short-rib polydactyly and Jeune syndromes are caused by mutations in WDR60. Am. J. Hum. Genet. 93, 515-523 (2013).

15. Dagoneau, N. et al. DYNC2H1 mutations cause asphyxiating thoracic dystrophy and short rib-polydactyly syndrome, type III. Am. J. Hum. Genet. 84, 706-711 (2009).

16. Merrill, A.E. et al. Ciliary abnormalities due to defects in the retrograde transport protein DYNC2H1 in short-rib polydactyly syndrome. Am. J. Hum. Genet. 84, 542-549 (2009).

17. Huber, C. \& Cormier-Daire, V. Ciliary disorder of the skeleton. Am. J. Med. Genet. C Semin Med. Genet. 160C, 165-174 (2012).

18. Halbritter, J. et al. Defects in the IFT-B component IFT172 cause Jeune and Mainzer-Saldino syndromes in humans. Am. J. Hum. Genet. 93, 915-925 (2013).

19. Tuz, K. et al. Mutations in CSPP1 cause primary cilia abnormalities and Joubert syndrome with or without Jeune asphyxiating thoracic dystrophy. Am. J. Hum. Genet. 94, 62-72 (2014).

20. Beales, P.L. et al. IFT80, which encodes a conserved intraflagellar transport protein, is mutated in Jeune asphyxiating thoracic dystrophy. Nat. Genet. 39, 727-729 (2007).

21. Perrault, I. et al. Mainzer-Saldino syndrome is a ciliopathy caused by IFT140 mutations. Am. J. Hum. Genet. 90, 864-870 (2012).

22. Bredrup, C. et al. Ciliopathies with skeletal anomalies and renal insufficiency due to mutations in the IFT-A gene WDR19. Am. J. Hum. Genet. 89, 634-643 (2011).

23. Davis, E.E. et al. TTC21B contributes both causal and modifying alleles across the ciliopathy spectrum. Nat. Genet. 43, 189-196 (2011).

24. Shaheen, R. et al. A founder CEP120 mutation in Jeune asphyxiating thoracic dystrophy expands the role of centriolar proteins in skeletal ciliopathies. Hum. Mol. Genet. 24, 1410-1419 (2014).

25. Miller, K.A. et al. Cauli: a mouse strain with an Ift140 mutation that results in a skeletal ciliopathy modelling Jeune syndrome. PLoS Genet. 9, e1003746 (2013).

26. Ocbina, P.J., Eggenschwiler, J.T., Moskowitz, I. \& Anderson, K.V. Complex interactions between genes controlling trafficking in primary cilia. Nat. Genet. 43, 547-553 (2011).

27. Schulte-Merker, S. \& Stainier, D.Y.R. Out with the old, in with the new: reassessing morpholino knockdowns in light of genome editing technology. Development 141, 3103-3104 (2014)

28. Kok, F.O. et al. Reverse genetic screening reveals poor correlation between morpholino-induced and mutant phenotypes in zebrafish. Dev. Cell 32, 97-108 (2015).

29. Tsujikawa, M. \& Malicki, J. Intraflagellar transport genes are essential for differentiation and survival of vertebrate sensory neurons. Neuron 42, 703-716 (2004).

30. Kramer-Zucker, A.G. et al. Cilia-driven fluid flow in the zebrafish pronephros, brain and Kupffer's vesicle is required for normal organogenesis. Development 132, 1907-1921 (2005).

31. Lunt, S.C., Haynes, T. \& Perkins, B.D. Zebrafish ift57, ift88, and ift172 intraflagellar transport mutants disrupt cilia but do not affect hedgehog signaling. Dev. Dyn. 238, 1744-1759 (2009).

32. Ryan, S. et al. Rapid identification of kidney cyst mutations by whole exome sequencing in zebrafish. Development 140, 4445-4451 (2013).
33. Sun, Z. et al. A genetic screen in zebrafish identifies cilia genes as a principal cause of cystic kidney. Development 131, 4085-4093 (2004).

34. Schmidts, M. et al. Exome sequencing identifies DYNC2H1 mutations as a common cause of asphyxiating thoracic dystrophy (Jeune syndrome) without major polydactyly, renal or retinal involvement. J. Med. Genet. 50, 309-323 (2013).

35. DiBella, L.M., Smith, E.F., Patel-King, R.S., Wakabayashi, K.-i. \& King, S.M A novel Tctex2-related light chain is required for stability of inner dynein arm I1 and motor function in the Chlamydomonas flagellum. J. Biol. Chem. 279, 21666-21676 (2004).

36. Pazour, G.J., Agrin, N., Leszyk, J. \& Witman, G.B. Proteomic analysis of a eukaryotic cilium. J. Cell Biol. 170, 103-113 (2005).

37. Yang, P. \& Sale, W.S. The Mr 140,000 intermediate chain of Chlamydomonas flagellar inner arm dynein is a WD-repeat protein implicated in dynein arm anchoring. Mol. Biol. Cell 9, 3335-3349 (1998).

38. El Hokayem, J. et al. NEK1 and DYNC2H1 are both involved in short rib polydactyly Majewski type but not in Beemer Langer cases. J. Med. Genet. 49, 227-233 (2012).

39. Baujat, G. et al. Asphyxiating thoracic dysplasia: clinical and molecular review of 39 families. J. Med. Genet. 50, 91-98 (2013).

40. Pazour, G.J. et al. Chlamydomonas IFT88 and its mouse homologue, polycystic kidney disease gene tg737, are required for assembly of cilia and flagella. J. Cell Biol. 151, 709-718 (2000).

41. Marszalek, J.R., Ruiz-Lozano, P., Roberts, E., Chien, K.R. \& Goldstein, L.S. Situs inversus and embryonic ciliary morphogenesis defects in mouse mutants lacking the KIF3A subunit of kinesin-II. Proc. Natl Acad. Sci. USA 96, 5043-5048 (1999).

42. Ashe, A. et al. Mutations in mouse Ift144 model the craniofacial, limb and rib defects in skeletal ciliopathies. Hum. Mol. Genet. 21, 1808-1823 (2012).

43. Pazour, G.J., Dickert, B.L. \& Witman, G.B. The DHC1b (DHC2) isoform of cytoplasmic dynein is required for flagellar assembly. J. Cell Biol. 144, 473-481 (1999).

44. Porter, M.E., Bower, R., Knott, J.A., Byrd, P. \& Dentler, W. Cytoplasmic dynein heavy chain $1 \mathrm{~b}$ is required for flagellar assembly in Chlamydomonas. Mol. Biol. Cell 10, 693-712 (1999).

45. Hou, Y., Pazour, G.J. \& Witman, G.B. A dynein light intermediate chain, D1bLIC, is required for retrograde intraflagellar transport. Mol. Biol. Cell 15, 4382-4394 (2004).

46. Iomini, C., Babaev-Khaimov, V., Sassaroli, M. \& Piperno, G. Protein particles in Chlamydomonas flagella undergo a transport cycle consisting of four phases. J. Cell Biol. 153, 13-24 (2001).

47. Engel, B.D. et al. The role of retrograde intraflagellar transport in flagellar assembly, maintenance, and function. J. Cell Biol. 199, 151-167 (2012).

48. Lin, H., Nauman, N.P., Albee, A.J., Hsu, S. \& Dutcher, S.K. New mutations in flagellar motors identified by whole genome sequencing in Chlamydomonas. Cilia 2, 14 (2013).

49. Asante, D., Stevenson, N.L. \& Stephens, D.J Subunit composition of the human cytoplasmic dynein-2 complex. J. Cell Sci. 127, 4774-4787 (2014).

50. Witman, G.B. in Dyneins: Structure, Biology and Disease. (ed. King, S.M.) 395-421 (Elsevier Inc., 2012).

51. King, S.M. in Dyneins: Structure, Biology and Disease. (ed. King, S.M.) 209-243 (Elsevier, Inc., 2012).

52. DiBella, L.M. et al. The Tctex1/Tctex2 class of dynein light chains. Dimerization, differential expression, and interaction with the LC8 protein family. J. Biol. Chem. 276, 14366-14373 (2001).

53. Goggolidou, P. et al. ATMIN is a transcriptional regulator of both lung morphogenesis and ciliogenesis. Development 141, 3966-3977 (2014)

54. Lee, J.E. \& Gleeson, J.G. A systems-biology approach to understanding the ciliopathy disorders. Genome Med. 3, 59 (2011).

55. Khanna, H. et al. A common allele in RPGRIP1L is a modifier of retinal degeneration in ciliopathies. Nat. Genet. 41, 739-745 (2009).

56. Kiesewetter, S. et al. A mutation in CFTR produces different phenotypes depending on chromosomal background. Nat. Genet. 5, 274-278 (1993).

57. Brown, J.M., Fine, N.A., Pandiyan, G., Thazhath, R. \& Gaertig, J. Hypoxia regulates assembly of cilia in suppressors of Tetrahymena lacking an intraflagellar transport subunit gene. Mol. Biol. Cell 14, 3192-3207 (2003).

58. Hou, Y. et al. Functional analysis of an individual IFT protein: IFT46 is required for transport of outer dynein arms into flagella. J. Cell Biol. 176, 653-665 (2007).

59. Craige, B. et al. CEP290 tethers flagellar transition zone microtubules to the membrane and regulates flagellar protein content. J. Cell Biol. 190, 927-940 (2010).

60. Smith, E.F. \& Lefebvre, P.A. PF16 encodes a protein with armadillo repeats and localizes to a single microtubule of the central apparatus in Chlamydomonas flagella. J. Cell Biol. 132, 359-370 (1996)

61. Wilkerson, C.G., King, S.M., Koutoulis, A., Pazour, G.J. \& Witman, G.B. The 78,000 M(r) intermediate chain of Chlamydomonas outer arm dynein isa 
WD-repeat protein required for arm assembly. J. Cell Biol. 129, 169-178 (1995).

62. Berthold, P., Schmitt, R. \& Mages, W. An engineered Streptomyces hygroscopicus aph 7 " gene mediates dominant resistance against hygromycin B in Chlamydomonas reinhardtii. Protist 153, 401-412 (2002).

63. Gorman, D.S. \& Levine, R.P. Cytochrome $\mathrm{f}$ and plastocyanin: their sequence in the photosynthetic electron transport chain of Chlamydomonas reinhardi. Proc. Natl Acad. Sci. USA 54, 1665-1669 (1965).

64. Sager, R. \& Granick, S. Nutritional studies with Chlamydomonas reinhardi. Ann N Y Acad. Sci. 56, 831-838 (1953).

65. Silflow, C.D. et al. The Vfl1 Protein in Chlamydomonas localizes in a rotationally asymmetric pattern at the distal ends of the basal bodies. J. Cell Biol. 153, 63-74 (2001).

66. Lechtreck, K.F., Luro, S., Awata, J. \& Witman, G.B. HA-tagging of putative flagellar proteins in Chlamydomonas reinhardtii identifies a novel protein of intraflagellar transport complex B. Cell Motil. Cytoskeleton 66 , 469-482 (2009).

67. Awata, J., Takada, S., Standley, C., Lechtreck, K.F., Bellvé, K.F., Pazour, G.J., Fogarty, K.E. \& Witman, G.B. Nephrocystin-4 controls ciliary trafficking of membrane and large soluble proteins at the transition zone. J. Cell Sci. 127, 4714-4727 (2014).

68. Witman, G.B., Carlson, K., Berliner, J. \& Rosenbaum, J.L. Chlamydomonas flagella. I. Isolation and electrophoretic analysis of microtubules, matrix, membranes, and mastigonemes. J. Cell Biol. 54, 507-539 (1972).

\section{Acknowledgements}

We thank Sarah Bond (Genetics and Genomics Medicine Unit, ICH, UCL) for assistance with Sanger Sequencing, Dr Elizabeth Smith (Dartmouth College) for providing Chlamydomonas strains, and Dr John Leszyk (UMMS Proteomics and Mass Spectrometry Facility) and Dr Bin Deng (Vermont Genetics Network Proteomics Facility, University of Vermont) for help with mass spectrometry. Andrew Phillips of HGMD (Cardiff) assisted in nomenclature for the TCTEX1D2 exon 1-2 mutation. The TCTEX1D2 SF-TAP construct was kindly provided by N. Katsanis and J. Willer. We are grateful to the UK10K consortium, in particular, the Rare Diseases Group for making this study possible; a full list of the UK10K investigators is available at http://www.uk10k.org/ publications_and_posters.html. Funding for UK10K was provided by the Wellcome Trust under award WT091310. This research was supported by National Institutes of Health (NIH) grants RO1 AR062651 and R01 AR066124 (to D.K.), R01 GM051293 (to S.M.K.) and R37 GM030626 (to G.B.W.), by the Robert W. Booth Endowment at UMMS (to G.B.W.) and by an NIH National Institute of General Medical Sciences Institutional Development Award (IDeA) P20 GM103449 (to the Vermont Genetics Network). C.A.J. acknowledges funding from a Sir Jules Thorn Award for Biomedical Research (JTA/09). W.H. was supported by a stipend from the Rosetree's Trust (grant A465). P.J.S. is funded by the British Heart Foundation. H.K. acknowledges funding from the Scientific and Technological Research Council of Turkey (TUBITAK, grant number 112S398) supported by the overall consortium, CRANIRARE-2, the European Research Area Network (ERA-Net) for research programmes on rare diseases (2011-2015). C.W. is supported by an Australian National Health and Medical Research Council grant (APP1045464) and a
University of Queensland (UQ) Vice Chancellor's Senior Research Fellowship, and C.R.C. by a UQ International PhD Scholarship. M.U. and K.B. acknowledge funding from FP7 grant agreement no. 278568, PRIMES. R.R. is supported by the Netherlands Organization for Scientific Research (NWO Vici-865.12.005). M.U., P.L.B., R.R. and C.A.J. acknowledge funding from the European Community's Seventh Framework Programme FP7/2009 under grant agreement no: 241955, SYSCILIA. M.S., P.L.B. and R.R. acknowledge funding from the Dutch Kidney Foundation (CP11.18). P.L.B. and H.M.M. are supported by the Great Ormond Street Hospital Children's Charity. M.S. is supported by an Action Medical Research UK Clinical Training Fellowship (RTF-1411), a Radboud Excellence Initiative and Radboud Hypatia Fellowship, and acknowledges funding from the German Research Foundation (DFG; Collaborative Research Center 1140, KIDGEM).

\section{Author contributions}

M.S., Y.H., H.M.M. and G.B.W. conceived the study and M.S., Y.H., S.M.K., C.W., R.R., H.M.M. and G.B.W. wrote the manuscript. M.S., Y.H., C.R.C., D.A.M., C.H., K.B., J.v.R. M.U., C.W., V.C.-D., R.R., H.M.M. and G.B.W. supervised experiments and were involved in data interpretations. M.S. and S.P.T. performed whole-exome sequencing analysis and M.S. performed zebrafish experiments and immunofluorescence analysis of human fibroblasts. Y.H. performed Chlamydomonas experiments. S.M.K. prepared antibodies against Chlamydomonas dynein light chains. C.R.C. performed immunoprecipitations of human TCTEX1D2. C.H. and J.-M.P. performed linkage analysis in human subjects. M.P., Z.M.Y. and W.H. performed Sanger Sequencing of human samples. D.A.M., J.v.R., S.E.C.v.B., S.J.F.L. and R.R. performed mammalian proteomics experiments and proteomic bioinformatics analysis. K.B. and M.U. performed mammalian proteomics mass spectrometry analysis. M.S., C.A.J., P.J.S., H.K., D.K., P.L.B. L.A.-G., V.C.-D. and H.M.M. were involved in patient enrolment. UK10K Consortium provided whole-exome sequencing data.

\section{Additional information}

Supplementary Information accompanies this paper at http://www.nature.com/ naturecommunications

Competing financial interests: The authors declare no competing financial interests.

Reprints and permission information is available online at http://npg.nature.com/ reprintsandpermissions/

How to cite this article: Schmidts, M. et al. TCTEX1D2 mutations underlie Jeune asphyxiating thoracic dystrophy with impaired retrograde intraflagellar transport. Nat. Commun. 6:7074 doi: 10.1038/ncomms8074 (2015).

This work is licensed under a Creative Commons Attribution 4.0 International License. The images or other third party material in this article are included in the article's Creative Commons license, unless indicated otherwise in the credit line; if the material is not included under the Creative Commons license, users will need to obtain permission from the license holder to reproduce the material. To view a copy of this license, visit http://creativecommons.org/licenses/by/4.0/

Saeed Al-Turki ${ }^{18,19}$, Carl Anderson ${ }^{18}$, Richard Anney ${ }^{20}$, Dinu Antony ${ }^{1}$, Jennifer Asimit ${ }^{18}$, Mohammad Ayub ${ }^{21}$, Jeff Barrett ${ }^{18}$, Inês Barroso ${ }^{18}$, Jamie Bentham ${ }^{22}$, Shoumo Bhattacharya ${ }^{22}$, Douglas Blackwood ${ }^{23}$, Martin Bobrow ${ }^{24}$, Elena Bochukova ${ }^{25}$, Patrick Bolton ${ }^{26}$, Chris Boustred ${ }^{27}$, Gerome Breen ${ }^{26,28}$, Marie-Jo Brion ${ }^{27}$, Andrew Brown ${ }^{18}$, Mattia Calissano ${ }^{29}$, Keren Carss ${ }^{18}$, Krishna Chatterjee ${ }^{25}$, Lu Chen ${ }^{18,30}$, Sebhattin Cirak ${ }^{29}$, Peter Clapham ${ }^{18}$, Gail Clement ${ }^{31}$, Guy Coates ${ }^{18}$, David Collier ${ }^{32,33}$, Catherine Cosgrove ${ }^{22}$, Tony Cox ${ }^{18}$, Nick Craddock ${ }^{34,35}$, Lucy Crooks ${ }^{18}$, Sarah Curran ${ }^{26}$, Allan Daly ${ }^{18}$, Petr Danecek ${ }^{18}$, George Davey Smith ${ }^{27}$, Aaron Day-Williams ${ }^{18,36}$, lan Day ${ }^{27}$, Richard Durbin ${ }^{18}$, Sarah Edkins ${ }^{18}$, Peter Ellis ${ }^{18}$, David Evans ${ }^{27}$, I Sadaf Farooqi ${ }^{25}$, Ghazaleh Fatemifar ${ }^{27}$, David Fitzpatrick ${ }^{37}$, Paul Flicek ${ }^{38}$, Jamie Floyd ${ }^{18}$, A Reghan Foley ${ }^{29}$, Chris Franklin ${ }^{18}$, Marta Futema ${ }^{39}$, Louise Gallagher ${ }^{19}$, Tom Gaunt ${ }^{27}$, Daniel Geschwind ${ }^{40}$, Celia Greenwood ${ }^{41,42}$, Detelina Grozeva ${ }^{24}$, Xiaosen Guo ${ }^{43}$, Hugh Gurling ${ }^{44}$, Deborah Hart ${ }^{31}$, Audrey Hendricks ${ }^{18}$, Peter Holmans ${ }^{34,35}$, Jie Huang ${ }^{18}$, Steve E. Humphries ${ }^{39}$, Matt Hurles ${ }^{18}$, Pirro Hysi ${ }^{31}$, David Jackson ${ }^{18}$, Yalda Jamshidi ${ }^{45}$, David Jewell ${ }^{27}$, Joyce Chris ${ }^{18}$, Jane Kaye ${ }^{46}$, Thomas Keane ${ }^{18}$, John Kemp ${ }^{27}$, Karen Kennedy ${ }^{18}$, Alastair Kent ${ }^{47}$, Anja Kolb-Kokocinski ${ }^{18}$, Genevieve Lachance ${ }^{31}$, Cordelia Langford ${ }^{18}$, Irene Lee ${ }^{48}$, Rui Lii, ${ }^{41,4}$, Yingrui Li ${ }^{43}$, 
Liu Ryan ${ }^{50}$, Jouko Lönnqvist ${ }^{51}$, Margarida Lopes ${ }^{18,52}$, Daniel G. MacArthur ${ }^{18,53,54}$, Mangino Massimo ${ }^{31}$, Jonathan Marchini ${ }^{55}$, John Maslen ${ }^{18}$, Shane McCarthy ${ }^{18}$, Peter McGuffin ${ }^{26}$, Andrew Mclntosh ${ }^{6}$, Andrew McKechanie ${ }^{6}$, Andrew McQuillin ${ }^{44}$, Yasin Memari ${ }^{18}$, Sarah Metrustry ${ }^{31}$, Josine Min ${ }^{27}$, Alireza Moayyeri ${ }^{31}$, James Morris ${ }^{18}$, Dawn Muddyman ${ }^{18}$, Francesco Muntoni ${ }^{29}$, Kate Northstone ${ }^{27}$, Michael O'Donovan ${ }^{34,35}$, Stephen O'Rahilly ${ }^{25}$, Alexandros Onoufriadis', Karim Oualkacha ${ }^{56}$, Michael Owen ${ }^{34,35}$, Aarno Palotie ${ }^{18,57}$, Kalliope Panoutsopoulou ${ }^{18}$, Victoria Parker ${ }^{25}$, Jeremy Parr ${ }^{58}$, Lavinia Paternoster ${ }^{27}$, Tiina Paunio ${ }^{51}$, Felicity Payne ${ }^{18}$, John Perry $31,52,59,60$, Olli Pietilainen ${ }^{18,51,57}$, Vincent Plagnol ${ }^{61}$, Michael A. Quail ${ }^{18}$, Lydia Quaye $^{31}$, Lucy Raymond ${ }^{24}$, Karola Rehnström ${ }^{18}$, J. Brent Richards $31,41,49$, Sue Ring ${ }^{27}$, Graham R. S. Ritchie ${ }^{18,38}$, David B. Savage ${ }^{25}$, Nadia Schoenmakers ${ }^{25}$, Robert K. Semple ${ }^{25}$, Eva Serra ${ }^{18}$, Hashem Shihab ${ }^{27}$, So-Youn Shin ${ }^{18}$, David Skuse ${ }^{48}$, Kerrin Small ${ }^{31}$, Carol Smee ${ }^{18}$, Artigas María Soler ${ }^{62}$, Nicole Soranzo ${ }^{18}$, Lorraine Southam ${ }^{18}$, Tim Spector ${ }^{31}$, Beate St Pourcain ${ }^{27}$, David St. Clair ${ }^{63}$, Jim Stalker ${ }^{18}$, Gabriela Surdulescu ${ }^{31}$, Jaana Suvisaari ${ }^{51}$, loanna Tachmazidou ${ }^{18}$, Jing Tian ${ }^{43}$, Nic Timpson²7, Martin Tobin ${ }^{62}$, Ana Valdes ${ }^{31}$, Margriet van Kogelenberg ${ }^{18}$, Parthiban Vijayarangakannan ${ }^{18}$, Louise Wain ${ }^{62}$, Klaudia Walter ${ }^{18}$, Jun Wang ${ }^{43}$, Kirsten Ward ${ }^{31}$, Ellie Wheeler ${ }^{18}$, Ros Whittall ${ }^{39}$, Hywel Williams ${ }^{34,35}$, Kathy Williamson ${ }^{37}$, Scott G. Wilson ${ }^{31,64,65}$, Kim Wong ${ }^{18}$, Tamieka Whyte ${ }^{29}$, Xu ChangJiang ${ }^{41}$, Eleftheria Zeggini ${ }^{18}$, Feng Zhang ${ }^{31} \&$ Hou-Feng Zheng 41,49

\footnotetext{
18 The Wellcome Trust Sanger Institute, Wellcome Trust Genome Campus, Hinxton CB10 1HH, Cambridge, UK. ${ }^{19}$ Department of Pathology, King Abdulaziz Medical City, Riyadh, Saudi Arabia. ${ }^{20}$ Department of Psychiatry, Trinity Centre for Health Sciences, St. James Hospital, James's Street, Dublin 8 , Ireland. ${ }^{21}$ Durham University School of Medicine, Pharmacy and Health, Wolfson Research Institute, Queen's Campus, Stockton-on-Tees TS17 6BH, UK.

22 Department of Cardiovascular Medicine and Wellcome Trust Centre for Human Genetics, Roosevelt Drive, Oxford OX3 7BN, UK. ${ }^{23}$ Division of Psychiatry, The University of Edinburgh, Royal Edinburgh Hospital, Edinburgh EH10 5HF, UK. ${ }^{24}$ Department of Medical Genetics, Cambridge Institute for Medical Research, University of Cambridge, Cambridge CB2 2XY, UK. ${ }^{25}$ University of Cambridge Metabolic Research Laboratories, and NIHR Cambridge Biomedical Research Centre, Institute of Metabolic Science, Addenbrooke's Hospital, Cambridge CB2 OQQ, UK. ${ }^{26}$ Institute of Psychiatry, Kings College London, 16 De Crespigny Park, London SE5 8AF, UK. ${ }^{27}$ MRC CAiTE Centre, School of Social and Community Medicine, University of Bristol, Oakfield House, Oakfield Grove, Clifton, Bristol BS8 2BN, UK. ${ }^{28}$ NIHR BRC for Mental Health, Institute of Psychiatry and SLaM NHS Trust, King's College London, 16 De Crespigny Park, London SE5 8AF, UK. ${ }^{29}$ Dubowitz Neuromuscular Centre, UCL Institute of child health \& Great Ormond Street Hospital, London WC1N 1EH, UK.

30 Department of Haematology, University of Cambridge, Long Road, Cambridge CB2 OPT, UK. ${ }^{31}$ The Department of Twin Research \& Genetic Epidemiology, King's College London, St Thomas' Campus, Lambeth Palace Road, London SE1 7EH, UK. ${ }^{2}$ Social, Genetic and Developmental Psychiatry Centre, Institute of Psychiatry, King's College London, Denmark Hill, London SE5 8AF, UK. ${ }^{33}$ Lilly Research Laboratories, Eli Lilly \& Co. Ltd., Erl Wood Manor, Sunninghill Road, Windlesham, Surrey, UK. ${ }^{34}$ MRC Centre for Neuropsychiatric Genetics \& Genomics, Institute of Psychological Medicine \& Clinical Neurosciences, School of Medicine, Cardiff University, Cardiff CF14 4XN. ${ }^{35}$ Bart's and the London School of Medicine and Dentistry, East Lindon NHS Foundation Trust, London, UK. ${ }^{36}$ Department of Translational Sciences, Biogen Idec, 14 Cambridge Center, Cambridge, MA 02142, USA. 37 MRC Human Genetics Unit, MRC Institute of Genetic and Molecular Medicine, at the University of Edinburgh, Western General Hospital, Edinburgh, EH4 2XU, UK. ${ }^{38}$ European Bioinformatics Institute, Wellcome Trust Genome Campus, Hinxton, Cambridge, CB10 1SD, UK. ${ }^{39}$ Cardiovascular Genetics, BHF Laboratories, Rayne Building, Institute Cardiovascular Sciences, University College London, London WC1E 6JJ, UK. ${ }^{40}$ UCLA David Geffen School of Medicine, Los Angeles, California, USA. ${ }^{41}$ Departments of Epidemiology, Biostatistics and Occupational Health, Lady Davis Institute, Jewish General Hospital, McGill University, Montreal, Quebec, Canada.

42 Department of Oncology, McGill University, Montreal, Quebec, Canada. ${ }^{43}$ BGI-Shenzhen, Shenzhen 518083, China. ${ }^{44}$ Molecular Psychiatry Laboratory, Mental Health Sciences Unit, University College London, 21 University St. Rockefeller Building, London WC1E 6BT, UK. ${ }^{45}$ Human Genetics Research Centre, St George's University of London, UK. ${ }^{46}$ HeLEX-Centre for Health, Law and Emerging Technologies, Department of Public Health, University of Oxford, Old Road Campus, Oxford, OX3 7LF, UK. ${ }^{47}$ Genetic Alliance UK, 4D Leroy House, 436 Essex Road, London N1 3QP, UK. ${ }^{48}$ Behavioural and Brain Sciences Unit, UCL Institute of Child Health, London, WC1N 1EH, UK. ${ }^{49}$ Departments of Medicine \& Human Genetics, Lady Davis Institute, Jewish General Hospital, McGill University, Montreal, Quebec, Canada.. ${ }^{50}$ BGI-Europe, London. ${ }^{51}$ National Institute for Health and Welfare (THL), Helsinki. ${ }^{52}$ Wellcome Trust Centre for Human Genetics, Roosevelt Drive, Oxford, OX3 7BN, UK. ${ }^{53}$ Analytic and Translational Genetics Unit, Massachusetts General Hospital, Boston MA 02113, USA. 54 Program in Medical and Population Genetics, Broad Institute of Harvard and MIT, Cambridge MA 02132, USA. ${ }^{55}$ Department of Statistics, University of Oxford, 1 South Parks Road, Oxford OX1 3TG, UK. ${ }^{56}$ Department of Mathematics, Université de Québec À Montréal, Montréal, Québec, Canada. 57 Institute for Molecular Medicine Finland (FIMM), University of Helsinki, Helsinki, Finland. ${ }^{58}$ Institute of Neuroscience, Henry Wellcome Building for Neuroecology, Newcastle University, Framlington Place, Newcastle upon Tyne, NE2 4HH, UK. ${ }^{59}$ Genetics of Complex Traits, Peninsula Medical School, University of Exeter, Exeter, UK. ${ }^{60}$ Center for Statistical Genetics, University of Michigan, Ann Arbor, Michigan, USA. ${ }^{61}$ University College London (UCL) Genetics Institute (UGI) Gower Street, London, WC1E 6BT, UK. ${ }^{2}$ Departments of Health Sciences and Genetics, University of Leicester, Leicester, UK. ${ }^{63}$ Institute of Medical Sciences, University of Aberdeen, AB25 2ZD, UK. ${ }^{64}$ School of Medicine and Pharmacology, University of Western Australia, Perth, WA, Australia. ${ }^{65}$ Department of Endocrinology and Diabetes, Sir Charles Gairdner Hospital, Nedlands, WA, Australia
} 


\section{Corrigendum: TCTEX1D2 mutations underlie Jeune asphyxiating thoracic dystrophy with impaired retrograde intraflagellar transport}

Miriam Schmidts, Yuqing Hou, Claudio R. Cortés, Dorus A. Mans, Celine Huber, Karsten Boldt, Mitali Patel, Jeroen van Reeuwijk, Jean-Marc Plaza, Sylvia E.C. van Beersum, Zhi Min Yap, Stef J.F. Letteboer, S. Paige Taylor, Warren Herridge, Colin A. Johnson, Peter J. Scambler, Marius Ueffing, Hulya Kayserili, Deborah Krakow, Stephen M. King, UK10K, Philip L. Beales, Lihadh AI-Gazali, Carol Wicking, Valerie Cormier-Daire, Ronald Roepman, Hannah M. Mitchison \& George B. Witman

Nature Communications 6:7074 doi:10.1038/ncomms8074 (2015); Published 05 June 2015; Updated 29 Mar 2016

The financial support for this article was not fully acknowledged. The Acknowledgements should have included the following:

PLB was supported by the National Institute for Health Research Biomedical Research Centre at Great Ormond Street Hospital for Children NHS Foundation Trust and University College London.

(c) (i) This work is licensed under a Creative Commons Attribution 4.0 International License. The images or other third party material in this article are included in the article's Creative Commons license, unless indicated otherwise in the credit line; if the material is not included under the Creative Commons license, users will need to obtain permission from the license holder to reproduce the material. To view a copy of this license, visit http://creativecommons.org/licenses/by/4.0/ 